\title{
Dissocier les mutilations génitales féminines de l'Islam
}

\author{
Ibrahim Lethome Asmani
}

Maryam Sheikh Abdi

Population Council

Follow this and additional works at: https://knowledgecommons.popcouncil.org/departments_sbsr-rh

Part of the Demography, Population, and Ecology Commons, Family, Life Course, and Society Commons, Gender and Sexuality Commons, International Public Health Commons, Medicine and Health Commons, Sociology of Culture Commons, and the Women's Health Commons How does access to this work benefit you? Let us know!

\section{Recommended Citation}

Lethome Asmani, Ibrahim and Maryam Sheikh Abdi. 2008. "Dissocier les mutilations génitales féminines de l'Islam." Nairobi: Population Council. 
Dissocier les mutilations génitales féminines de I'Islam

USAID

Population Council FRONTIERS 


\section{Dissocier les mutilations génitales féminines de l'Islam}

Ibrahim Lethome Asmani

Maryam Sheikh Abdi

2008

La présente publication a pu être réalisée grâce au généreux soutien du peuple américain, par l'intermédiaire de l'Agence américaine pour le développement international (United States Agency for International Development, USAID) en vertu des termes de l'accord de coopération $\mathrm{n}^{\circ} \mathrm{HRN}-\mathrm{A}-00-98-00012-00$, projet interne 80165309. Ses contenus relèvent de la responsabilité du programme FRONTIERS et ne reflètent pas nécessairement l'opinion de l'USAID ou du gouvernement des États-Unis d'Amérique. 


\section{TABLE DES MATIÈRES}

Remerciements

Contexte

Pourquoi les MGF sont-elles associées à l'Islam ?

Termes religieux utilisés pour désigner la pratique

Le mot khitaan

L'interprétation des textes

La croyance selon laquelle les MGF garantissent la chasteté

L'aptitude au mariage

La croyance selon laquelle les MGF assurent la propreté rituelle (tohara)

L'Islam et les MGF

Quand un acte est-il considéré comme islamique?

Les enseignements islamiques qui s'opposent aux MGF

Les MGF sont contraires aux objectifs de la charia

Allah (SWT) condamne ceux qui modifient Sa création

Causer un préjudice est illégal

L'Islam condamne les pratiques culturelles néfastes

Les femmes ont droit à un corps sain et au plaisir sexuel dans leurs relations

conjugales

L'infibulation affecte la propreté rituelle (tohara)

Les MGF sont une violation des droits humains des femmes et des jeunes filles

Des efforts sont nécessaires pour corriger les maux dans la communauté

L'origine des MGF n'est pas islamique

\section{Conclusions}

Glossaire des concepts/termes 


\section{REMERCIEMENTS}

La présente brochure a été rédigée avec l'aide de nombreuses personnes dont les rôles importants méritent d'être reconnus et appréciés.

Nous souhaitons remercier particulièrement Ian Askew, Stéphanie Joyce, Janet Munyasya, Francis Onyango et Adam Isaac, tous membres de Population Council, pour leurs précieuses contributions tout au long du processus de rédaction. Nous adressons également nos chaleureux remerciements à Cheikh Khalfan Khamis, président du Conseil des savants musulmans du Kenya (Council of Scholars of Kenya, Majlis-ulamaa), pour ses contributions durant les phases initiales et son assistance tout au long du processus, ainsi qu'à Rahma Abdi Digale pour son excellent travail de dactylographie de la version arabe à l'origine des traductions anglaise et française de la présente brochure.

Nous avons grandement bénéficié des diverses réunions des savants musulmans de la province nord-orientale et d'autres régions du Kenya organisées par le bureau de Population Council à Nairobi, ainsi que des séances de formation communautaires tenues à Wajir. Les opinions et les perceptions exprimées lors de ces évènements ont éclairé les questions cruciales abordées et analysées dans cette brochure.

Certaines des séances avec les savants ont été organisées en collaboration avec UNICEF/Kenya, FNUAP/Kenya, GTZ/Kenya, ainsi que le Conseil des imams et prédicateurs du Kenya (Council of Imams and Preachers of Kenya, CIPK) et nous leur sommes très reconnaissants pour leur coopération. Les enseignements tirés de ces partenariats ont considérablement contribué à la rédaction de la présente brochure. Zeinab A. Ahmed, du bureau d'UNICEF/Kenya à Garissa, a apporté de précieuses modifications à la brochure et ses efforts ont été très appréciés.

Les auteurs tiennent à remercier également l'USAID/Kenya pour son appui financier à la préparation du présent document.

Nous espérons sincèrement que cette brochure contribuera significativement à clarifier la position islamique correcte à l'égard des mutilations génitales féminines (MGF), aussi appelées excision, et qu'elle sera utile aux lecteurs pour prendre des décisions en connaissance de cause en vue d'abandonner la pratique. 


\section{CONTEXTE}

Les mutilations génitales féminines (MGF), aussi appelées excision, sont pratiquées dans au moins 28 pays d'Afrique subsaharienne, dans quelques pays du Moyen-Orient et d'Asie et parmi les populations immigrées de ces pays en Europe, Amérique du Nord et Australie. Au niveau mondial, on estime que 100 à 140 millions de jeunes filles et de femmes ont subi l'intervention, et qu'au moins trois millions de jeunes filles risquent d'y être soumises chaque année.

Les MGF comprennent toutes les procédures impliquant une ablation partielle ou totale des organes génitaux féminins externes à des fins non thérapeutiques ${ }^{1}$. L'Organisation mondiale de la santé (OMS) reconnaît quatre types de MGF, le plus extrême étant le type III (infibulation $^{2}$ ), parfois dénommé circoncision pharaonique ${ }^{3}$; environ 15 pour cent de toutes les formes de mutilations génitales féminines appartiennent à cette catégorie ${ }^{4}$.

La communauté ethnique somalienne, au Kenya comme en Somalie, à Djibouti et en Éthiopie, pratique l'excision génitale féminine depuis des siècles et la coutume semble être restée en grande partie inchangée jusqu'à nos jours. En 2003, l'enquête démographique et de santé au Kenya (KDHS $)^{5}$ a constaté une prévalence de 96,8 pour cent parmi les Somali ayant participé à l'enquête et de 98,9 pour cent dans la province nord-orientale où vivent la majorité des Somali. Cette communauté pratique essentiellement les MGF du type III ou infibulation.

Le programme FRONTIERS de santé reproductive du Population Council, appuyé par l'USAID/Kenya, a réalisé deux études pour mieux comprendre la pratique des MGF chez les Somali de la province nord-orientale. Leur but était de fournir les informations nécessaires à la conception et à la mise en place d'interventions en vue d'encourager l'abandon de la pratique $^{6}$. La première étude de diagnostic a été réalisée dans les districts de Mandera et Wajir ainsi que dans le quartier Eastleigh de Nairobi en 2004. La seconde, une étude de base, a été réalisée en novembre 2005 dans six localités de la division centrale du district de Wajir. Les deux études ont collecté des données au moyen d'interviews approfondies et de discussions de groupe faisant intervenir des leaders religieux et communautaires ainsi que des hommes et des femmes récemment mariés ou célibataires. La première étude comprenait également des interviews avec des prestataires de soins de santé et des clientes des services prénataux qui avaient été excisées et a examiné la disposition des cliniques à proposer des services de maternité sûrs.

Ces études ont confirmé que les MGF sont une pratique profondément enracinée et largement appuyée, soutenue par bon nombre de justifications culturelles qui renforcent sa persistance. Les trois principales raisons citées étaient les suivantes : les MGF sont une tradition somali,

\footnotetext{
${ }^{1}$ OMS. 2008. Éliminer les mutilations sexuelles féminines. Déclaration interinstitutions. OMS : Genève.

${ }^{2}$ Ibid.

${ }^{3}$ Le terme est employé pour décrire cette forme d'excision génitale car elle est supposée avoir été introduite par les Pharaons d'Égypte.

${ }^{4}$ Yoder PS et Khan S. 2007. Numbers of Women Circumcised in Africa: The Production of a Total. Calverton, Macro International Inc.

${ }^{5}$ Central Bureau of Statistics (CBS) [Kenya], Ministry of Health (MOH) [Kenya] et ORC Macro. 2004. Kenya Demographic and Health Survey 2003 (enquête démographique et de santé 2003 au Kenya). Calverton, Maryland : CBS, MOH, et ORC Macro.

${ }^{6}$ Jaldesa G. W., Askew I., Njue C., Wanjiru M.. 2004. Female genital cutting among the Somali of Kenya and management of its complications, Population Council: Nairobi, Kenya et FGM Baseline Study, Wajir District, 2005.
} 
elles sont prescrites par l'Islam et elles renforcent la valeur culturelle de la pureté sexuelle des femmes en contrôlant leur désir sexuel, ce qui permet de garantir la virginité avant le mariage et la fidélité tout au long de la vie d'une femme. Les études ont montré l'existence d'une crainte que les femmes ne «deviennent sauvages » et de mœurs faciles lorsqu'elles ne sont pas excisées et infibulées. Les MGF sont considérées à tort comme une manière de se conformer aux préceptes islamiques de chasteté et de moralité, et elles sont supposées renforcer la propreté rituelle des femmes, qui est une condition indispensable pour pouvoir prier.

Le précepte islamique ainsi perçu est un des principaux arguments avancés pour justifier les MGF dans les communautés musulmanes pratiquantes comme celles des Somali. Par conséquent, il apparaît nécessaire de vérifier la vraie position de l'Islam à l'égard de la pratique et de la communiquer aux leaders religieux des communautés islamiques qui pratiquent les MGF. La présente brochure est un humble effort pour clarifier la vérité au sujet de l'Islam et des pratiques de MGF, en procédant à un examen critique des éléments de preuve cités par les partisans de la pratique, notamment ceux qui la décrivent comme islamique.

\section{POURQUOI LES MGF SONT-ELLES ASSOCIÉES À L'ISLAM}

De nombreuses raisons sont invoquées pour justifier la pratique des MGF dans différentes communautés. Cependant, dans les communautés à prédominance musulmane, elle a été associée à l'Islam, et l'on croit fermement que toute femme musulmane doit y être soumise. Voici par exemple les opinions exprimées par la communauté somali de Wajir :

"Une femme non excisée n'est pas une musulmane, et même ses parents sont considérés comme étrangers à la religion, c'est ainsi que nous voyons les choses en tant que Somali », (hommes mariés, Wagberi) ${ }^{7}$.

Les raisons expliquant pourquoi la pratique a été associée à l'Islam sont examinées ci-après.

\section{Termes religieux utilisés pour désigner la pratique}

L'emploi de certains termes religieux pour désigner la pratique a donné à celle-ci une identité islamique et renforcé la croyance selon laquelle les MGF seraient prescrites par l'Islam. Un exemple est l'emploi du mot sunna, qui est un terme religieux islamique (voir l'encadré cidessous pour la définition de ce terme). Cette croyance est fortement ancrée dans la communauté somali :

«D'après la religion, le firouni (infibulation) n'est pas obligatoire tandis que la sunna est obligatoire » (hommes mariés). ${ }^{8}$

«L'Islam dit de couper juste un peu, c'est la sunna... »(hommes mariés, Wagberi) ${ }^{9}$.

D'autres termes islamiques tels que mandoub, mubaah et mashru'u sont également employés pour caractériser la pratique. Mandoub désigne un acte permis, qu'il est préférable d'accomplir plutôt que d'omettre. Mubaah désigne tout acte autorisé par l'Islam, mais ne

\footnotetext{
${ }^{7}$ FRONTIERS 2007, FGM/C Baseline Study in Wajir District.

${ }^{8}$ Ibid.

${ }^{9}$ Ibid.
} 
constituant ni une vertu ni un péché s'il est commis ou omis. Mashru'u est dérivé de la charia $^{10}$ et suggère que la pratique repose sur une base juridique islamique.

Les MGF ont également été dénommées tohara (propreté rituelle) en arabe et en swahili ${ }^{11}$ et dhahara en somali. La communauté somali emploie aussi des termes comme xalaalin (rendre légal) et Islaamin (islamiser) pour souligner son caractère islamique. Les arabophones qualifient les femmes excisées de mutoharat (lavées ou purifiées) et les femmes non excisées de ghulfa' $a$ (souillées ou impures) ${ }^{12}$.

\section{Définition du terme sunna}

1. Littéralement, en arabe, « un chemin ou une voie ».

2. Dans le contexte religieux islamique : «mode de vie ou tradition du prophète Mahomet (PSL), c'est-à-dire ce qu'il a dit, ce qu'il a fait et ce qu'il a approuvé et qu'il est recommandé aux musulmans de pratiquer ».

3. Dans le fiqh, c'est-à-dire la jurisprudence islamique : « un acte facultatif qui est recommandé. S'il est accompli, la personne est récompensée, s'il n'est pas accompli la personne n'a pas péché ».

Dans le contexte des MGF, sunna signifie suivre la voie du prophète Mahomet (PSL). Les partisans de la pratique croient que les MGF faisaient partie de ses traditions. Ils croient aussi que la pratique est facultative et que le fait de l'observer confère de la vertu.

Ce mot est, par ailleurs, couramment utilisé dans un autre sens dans de nombreuses communautés musulmanes, à savoir pour désigner quelque chose de petit. Il est par exemple fréquent qu'une personne invite une autre à prendre un verre ou un casse-croûte en lui disant : «prenons une sunna » ce qui signifie «prenons un petit quelque chose $»$. Dans le contexte des MGF, l'emploi du terme sunna par les partisans de la pratique sous-entend le fait qu'il faut exciser une petite partie des organes génitaux féminins. Les personnes interrogées à Wajir sur ce que disait l'Islam de la pratique ont répondu ceci :

«...il dit de faire sunna, juste un saignement, il ne faut pas couper de chair ou alors juste un petit peu » (femmes mariées, Jogbaru).

\section{Le mot khitaan}

Les partisans des MGF ont traduit le mot arabe khitaan, qui apparaît dans de nombreux textes religieux, comme désignant à la fois la circoncision masculine et les MGF. Or, le terme ne décrit en réalité que la circoncision masculine ; les MGF sont en fait appelées khifaadh. Cependant, chaque fois que khitaan apparaît dans un texte religieux, les partisans s'y réfèrent pour justifier un fondement islamique des MGF.

\section{L'interprétation des textes}

Les partisans des MGF utilisent des interprétations erronées de certains versets du Coran pour donner une base islamique à la pratique. Par exemple « ...puis Nous t'avons révélé [Oh Mahomet] : "suis la religion d'Abraham" » (Coran $16: 123)$. Ce verset ordonne au prophète Mahomet (PSL) ${ }^{13}$, et donc à tous les musulmans, d'imiter le mode de vie du prophète Abraham $(\mathrm{AS})^{14}$. La croyance veut que parce que le prophète Abraham (AS) a été circoncis à l'âge de 80 ans, tous les musulmans doivent être circoncis. Ce verset fera l'objet d'une analyse objective au chapitre consacré à l'Islam et les MGF afin de vérifier s'il peut être utilisé pour justifier ces dernières.

\footnotetext{
${ }^{10}$ Système juridique islamique.

${ }^{11}$ À présent, c'est le terme de kukeketa qui est utilisé en swahili pour décrire la mutilation causée par la pratique.

${ }^{12}$ Cheikh Ali Hashim as-Siraj, (2002), Female Genital Mutilation is Female Infanticide (traduction), p. 31.

${ }^{13}$ Paix soit sur lui.

${ }^{14}$ Alleihi Salaam (que la paix soit avec lui) : voir le glossaire des termes.
} 
Les partisans de la pratique fondent aussi leurs arguments sur certains ahadith $^{15}$ dans lesquels le prophète Mahomet (PSL) aurait donné instruction aux musulmans, hommes et femmes, d'être circoncis. Ces ahadith seront également analysés au chapitre concernant l'Islam et les MGF afin de déterminer leur authenticité et leur pertinence en tant que fondements des MGF.

\section{La croyance selon laquelle les MGF garantissent la chasteté}

La chasteté est une vertu très importante dans l'Islam et les musulmans sont tenus d'être chastes et de faire preuve d'une grande droiture morale. Les partisans des MGF croient que les femmes sont animées par de puissantes pulsions sexuelles (ghilma) et que si leurs organes génitaux ne sont pas excisés leur sexualité sera incontrôlable. Ils croient aussi que le clitoris et les petites lèvres vont s'allonger et rendre la femme sexuellement hyperactive. Ainsi, l'ablation partielle ou totale de ces organes est recommandée afin d'assurer que les femmes restent chastes tout au long de leur vie. De nombreux musulmans croient que cette recommandation est conforme aux enseignements islamiques sur la chasteté.

Un praticien traditionnel a affirmé qu'une jeune fille ou une femme non excisée « commencera à courir après les hommes à cause de sa sensualité incontrôlable, de son désir sexuel excessif, elle sera très vulnérable, elle n'aura aucune sécurité et finira par perdre son honneur. Les jeunes filles excisées ne chercheront pas un autre homme...» (exciseur traditionnel, discussions en groupes de réflexion, $2004^{16}$ ).

\section{L'aptitude au mariage}

Le mariage est un acte recommandé par l'Islam et considéré comme une sunna importante du prophète Mahomet (PSL) qui a dit : «le mariage est ma sunna et quiconque se détourne de ma sunna ne fait pas partie des miens ${ }^{17} »$. Les MGF sont étroitement associées à l'aptitude d'une jeune fille ou d'une femme au mariage ; en effet, on considère que les filles excisées, sont chastes, que leurs désirs sexuels sont contrôlés et que leur virginité est garantie, ce qui les rend aptes au mariage. Dans la communauté somali, la croyance veut que l'infibulation (type III) assure la virginité, de sorte qu'une femme ou une jeune fille non infibulée n'est pas considérée comme vierge. L'infibulation est vue à la fois comme un moyen de préserver la virginité et un signe de virginité. Les hommes craignent qu'une jeune fille non excisée et non infibulée ne soit pas une partenaire attrayante pour le mariage.

«Si la jeune fille n'est pas excisée, quelle que soit sa beauté, elle n'est pas désirée par les hommes. Il [1'homme] veut ou préfère celle qui est cousue, avec laquelle il sera heureux de passer ses nuits. Mais il ne sera pas heureux avec celle qui est ouverte. Je ferai tout ce qu'il faut pour qu'ils aiment ma fille ; je vais la coudre bien serré » (hommes âgés, discussions en groupes de réflexion ${ }^{18}$ ).

\section{La croyance selon laquelle les MGF assurent la propreté rituelle (tohara)}

Dans l'Islam, la purification rituelle (tohara) est très importante et aucun acte de culte n'est acceptable si une personne n'est pas en état de propreté. Selon une croyance très tenace, les femmes non excisées ne peuvent pas atteindre cet état de propreté parce que leur clitoris s'allonge et forme des plis cutanés qui abritent des souillures difficiles à enlever.

\footnotetext{
${ }^{15}$ Paroles du prophète Mahomet (PSL). Ahadith est le pluriel de hadith.

${ }^{16}$ Jaldesa G. W., Askew I., Njue C., Wanjiru M. Female genital cutting among the Somali of Kenya and management of its complications. Population Council : Nairobi, Kenya

${ }^{17}$ Sahih Boukhari, chapitre sur nikaah (le mariage), hadith $\mathrm{n}^{\circ} 5063$.

${ }^{18}$ Jaldesa G. W., Askew I., Njue C., Wanjiru M. Female genital cutting among the Somali of Kenya and management of its complications. Population Council : Nairobi, Kenya
} 
«Celles qui ne sont pas excisées sont sales, elles produiront toujours une odeur fétide, alors nous sommes excisées pour rester propres » (femmes récemment mariées, discussions en groupes de réflexion ${ }^{19}$ ).

Pour les raisons exposées ci-dessus, les MGF ont été considérées pendant longtemps comme une pratique islamique. Aussi est-il impératif d'examiner ce que disent réellement les textes islamiques à leur sujet et de vérifier si elles constituent ou non une pratique islamique.

\section{L'ISLAM ET LES MGF}

L'Islam est un mode de vie complet et ses enseignements régissent tous les aspects de la vie d'un musulman. Les fidèles sont tenus de se conformer à l'Islam et d'appliquer ses enseignements dans leurs vies quotidiennes. Puisque l'Islam donne des orientations couvrant tous les aspects de la vie humaine, les MGF en font partie également, et il convient de consulter les sources authentiques pour savoir si celles-ci constituent ou non une pratique religieuse.

\section{Quand un acte est-il considéré comme islamique ?}

Tous les actes accomplis au nom de l'Islam ne sont pas nécessairement islamiques. Bon nombre d'actions sont entreprises pour des motifs purement culturels, mais avec le temps, elles peuvent acquérir une justification islamique, notamment au sein des communautés à prédominance musulmane. Cependant, un acte n'est considéré comme islamique que s'il s'appuie sur l'une des sources fondamentales de la pensée islamique. Ces sources sont les suivantes :

- le Saint Coran (les paroles divines d'Allah-SWT ${ }^{20}$ );

- la Sunna (ensemble des actes, paroles, déclarations ou approbations du prophète Mahomet (PSL) ;

- l'Ijma'a (consensus des savants de la religion);

- le Qiyas (déduction analogique).

Comme pour toute autre question nécessitant un verdict islamique clair, il est nécessaire de se référer à ces sources fondamentales de la jurisprudence islamique (fiqh) pour clarifier les fondements de la pratique des MGF. Il est indispensable de déterminer s'il existe des versets du Coran susceptibles de servir de preuve en faveur des MGF. De même, il convient de vérifier dans les traditions (sunna) du prophète (PSL), qui sont bien documentées, s'il existe ou non des traditions authentiques ou pertinentes pouvant servir à étayer la pratique. Il importe aussi de déterminer si les savants musulmans se sont déjà accordés sur une opinion considérant la pratique de l'excision comme conforme aux orientations du Coran et à la tradition authentique du prophète (PSL). Enfin, nous devrions être en mesure d'appliquer le principe du qiyas, ou de la déduction par analogie, pour établir les fondements des MGF.

\section{Le Coran}

Une analyse rigoureuse et objective du Coran révèle qu'il n'existe pas un seul verset préconisant les MGF. Cependant, certains versets sont utilisés par les partisans de la pratique pour étayer leur point de vue. Le verset $16: 123$ du Coran énonce par exemple : « ... suis la

\footnotetext{
19 Ibid.

${ }^{20}$ Subhanahu wa taala (le Très-Haut) : voir glossaire.
} 
religion [milat] d'Abraham ». Il exhorte les musulmans à faire tout ce qu'a fait le prophète Abraham (AS), y compris la circoncision masculine, parmi beaucoup d'autres actions qui font partie de sa milat. Cependant, dans le contexte qui nous occupe, ce verset ne s'applique qu'à la circoncision masculine puisque c'est un fait bien connu que le prophète Abraham (AS) a été circoncis à l'âge de 80 ans. Dans un hadith raconté par Abu Huraira $\left(\mathrm{RA}^{21}\right)$, le prophète Mahomet (PSL) a dit : "Abraham, l'ami d'Allah, a été circoncis à l'âge de 80 ans. Et il a été circoncis à Al-Qaddum » (Boukhari et Muslim) ${ }^{22}$.

Rien n'indique que l'une ou l'autre des deux épouses du prophète Abraham (AS), Sarah et Hajar, étaient excisées, donc aucun fait à l'appui des MGF, bien que les partisans de la pratique croient que Hajar ait été excisée par Sarah. Il est allégué que lorsque Hajar a conçu un enfant, Sarah a été jalouse et a juré de la tuer, mais que le prophète Abraham lui a conseillé de percer les oreilles d'Hajar et d'exciser une partie de ses organes génitaux pour annuler le serment. Cette allégation est dépourvue de fondement et constitue un simple mythe. Même en la supposant véridique, Hajar aurait alors subi l'excision comme une punition, et non comme un acte vertueux ou une tradition ${ }^{23}$. Cette histoire n'évoque pas non plus une obligation religieuse, ni une pratique courante car rien ne prouve que Sarah fût elle-même excisée.

En conclusion, il n'existe aucun verset dans le Coran susceptible d'être employé comme preuve en faveur de cette pratique. Au contraire, de nombreux versets condamnent sévèrement toute action altérant le corps humain de quelque manière que ce soit et interférant dans la création d'Allah (SWT) sans justification. Les exemples sont notamment : «...pas de changement à la création d'Allah. Voilà la religion de droiture; mais la plupart des gens ne savent pas »(Coran : $30: 30)$ et «...et ne vous jetez pas par vos propres mains dans la destruction $\gg($ Coran : $2: 195)$

\section{La Sunna}

Allah (SWT) a ordonné aux musulmans de suivre le prophète (PSL) car il constitue pour eux le meilleur exemple. Allah (SWT) a dit : "En effet, vous avez dans le messager d'Allah un excellent modèle [à suivre]... »(Coran : $33: 21)$. La sunna désigne l'ensemble des traditions et éléments du mode de vie du prophète Mahomet (PSL) que les musulmans sont tenus d'observer. S'il existe des faits prouvant que le prophète a réellement pratiqué les MGF, ou que celles-ci étaient pratiquées au cours de sa vie et qu'il les a approuvées, la pratique devient alors une sunna, une tradition à observer par les musulmans, soit comme obligation, soit à titre facultatif.

Il existe trois catégories de sunna:

Les approbations du prophète Mahomet (PSL) : Il s'agit de tout ce qui a été dit ou fait en sa présence et qu'il a approuvé, soit expressément soit implicitement. Pour ce qui est des MGF, il est important de déterminer si elles ont été pratiquées en sa présence et s'il les a approuvées pour qu'elles deviennent une tradition à suivre.

Il n'existe aucune preuve, dans aucune des traditions authentiques, confirmant que le prophète aurait approuvé les MGF. Il est attesté que l'un de ses compagnons, Ibn Abbass (RA), était circoncis, ce qui indique qu'il approuvait la circoncision masculine. Dans un récit de Said bin Jubeir, Ibn Abbass a été interrogé sur l'âge qu'il avait au moment de la mort du prophète

\footnotetext{
${ }^{21}$ Radi-Allahu Anhu (que Allah soit satisfait de lui) : voir glossaire des termes.

${ }^{22}$ Voir Nail-al-autwar, vol. 1, p. 181.

${ }^{23}$ Voir Ch. Ali Hashim as-Siraj, (2002), FGM/C is female infanticide, p. 19.
} 
(PSL) et il a dit : «j'étais circoncis à cette époque »(Al-Boukhari) ${ }^{24}$. Il est rapporté, par ailleurs, que le prophète ordonnait aux hommes embrassant l'Islam d'être circoncis, mais ne demandait pas aux femmes de faire de même. Dans un hadith raconté par Abu Huraira (RA), le prophète (PSL) a dit : «lorsqu'un homme devient un Musulman, il doit être circoncis ${ }^{25}$.

Les apologistes des MGF en tant que pratique islamique avancent qu'en l'absence de désapprobation explicite de la part du prophète (PSL), l'acte est autorisé (mubaah), ce qui lui donne un fondement islamique. En réponse à cela, il est important de rappeler que la règle générale concernant le corps humain est qu'aucune intervention ne peut être pratiquée sur lui sans preuve manifeste de sa justification. Ce principe est dérivé du Coran, qui affirme catégoriquement que le fait de blesser un organe humain sans motif est punissable par un acte comparable, afin d'en décourager d'autres. Allah (SWT) a dit : « ... et nous y avons prescrit pour eux vie pour vie, ceil pour ceil, nez pour nez, oreille pour oreille, dent pour dent. Les blessures tombent sous la loi du talion » (Coran : 5 : 45). Dans le sermon prononcé lors de son dernier pèlerinage (hijjatul widaa), le prophète Mahomet (PSL) a également été très explicite quant au caractère sacré du corps humain et souligné qu'il ne devait pas être endommagé sans justification.

Les actes du prophète Mahomet (PSL) : Il s'agit des actes qu'il a réellement posés, et que les Musulmans sont tenus de suivre comme exemple. Dans le contexte des MGF, il est important de déterminer si les femmes de son foyer, ou celles de ses compagnons, étaient ou non excisées. La biographie (sira) du prophète (PSL) a été rigoureusement compilée et exactement documentée. Cependant, elle ne fournit aucune preuve que les femmes de son foyer ou celles de ses compagnons étaient excisées. D'autre part, il est mentionné que ses deux petits-fils, Al-Hassan et Al-Hussein, ont été circoncis à l'âge de sept jours. Dans un hadith raconté par Aïcha $\left(\mathrm{RA}^{26}\right)$, l'une des épouses du prophète Mahomet (PSL), elle a dit : «le prophète a circoncis Al-Hassan et Al-Hussein le septième jour après leur naissance ${ }^{27}$. Mais il n'existe aucune preuve relative aux MGF dans ses actes.

Les paroles du prophète Mahomet (PSL) : Toute parole prononcée par le prophète (PSL) sous l'inspiration d'Allah (SWT) et ayant des implications religieuses fait partie de la sunna du prophète (PSL). De nombreuses pratiques religieuses ont été introduites par les paroles du prophète (PSL). Allah (SWT) a dit à ce sujet : "...et il ne prononce rien sous l'effet de [sa propre] passion » (Coran : $53: 3$ ) et «...ce n'est rien d'autre qu'une révélation inspirée » (Coran : $53: 4)$.

Les partisans des MGF emploient certains ahadith (paroles) du prophète (PSL) pour conférer un fondement islamique à la pratique et notamment à l'excision dite sunna. Cependant, toutes les paroles attribuées au prophète (PSL) ne sont pas à prendre au pied de la lettre, mais doivent être vérifiées afin d'en déterminer l'authenticité. Les savants spécialisés dans les paroles du prophète (PSL) (ulumm-alhadith) ont pu identifier et différencier les paroles qui sont authentiques (sahih) et celles qui ne le sont pas. Ces savants examinent notamment le contenu (matn) des hadiths et la chaîne des transmetteurs ${ }^{28}$ (sanad) afin d'en vérifier l'authenticité. Nous procédons ci-après à une analyse critique de ces ahadith afin de vérifier s'ils peuvent ou non être employés comme preuve du bien-fondé des MGF dans l'Islam.

\footnotetext{
${ }^{24}$ Voir Nail-al-autwar, vol. 1, p. 185.

${ }^{25}$ Al-Hafidh, At-talkhis (le résumé)

${ }^{26}$ Radi-Allahu Anha : que Allah soit satisfait d'elle : voir glossaire des termes.

${ }_{27}$ Al-Baihaqqy dans son livre Sunan-al-kubra (les principales traditions), vol. 9, p. 299 et 303.

${ }^{28}$ Ceux qui ont reçu le hadith du prophète (PSL) et l'ont transmis au compilateur.
} 


\section{Hadith d'Umm-Attiya}

C'est le hadith le plus communément utilisé pour associer les MGF à l'Islam. Il raconte que le prophète (PSL) a dit à une femme de Médine appelée Umm-Attiyah : «Oh Umm Attiyyah, "ashimmi" ${ }^{29}$ et n'exagère pas ; car agir ainsi préservera la beauté du visage de la femme et contentera le mari ». Le mot «ashimmi » a plusieurs significations, notamment « masser avec quelque chose de doux comme l'huile », « garder quelque chose levé », « embrasser », « sentir mauvais » ou «poser une chose par-dessus une autre ». Cependant, les partisans des MGF pensent que le mot veut dire « exciser une petite partie du clitoris », alors qu'il n'a pas cette signification.

Il existe plusieurs versions de ce hadith, mais toutes ont été déclarées dhaeef (faibles) car la chaîne des transmetteurs (sanad) ${ }^{30}$ est peu fiable et sa signification est controversée. Compte tenu des différents sens du mot «ashimmi », ce que le prophète (PSL) a voulu dire à la femme n'est pas clair. Néanmoins, l'excision génitale féminine ne faisait pas partie de son conseil puisque le mot employé n'a pas cette signification.

En règle générale, aucun hadith faible ne peut être utilisé pour justifier quoi que ce soit dans l'Islam, notamment en ce qui concerne le corps humain. Un éminent spécialiste de la jurisprudence islamique (fiqh), Ash-Shaukany, conclut dans son livre Nail-al-autwar que le hadith ne peut être employé comme fondement des MGF. Même certains partisans de la pratique reconnaissent que ce hadith d'Umm-Attiyah ne peut être utilisé seul pour justifier les MGF ; mais ils affirment que d'autres ahadith peuvent être employés pour le corroborer et élever ainsi son statut pour remédier à sa faiblesse. Cependant, des experts en ahadith ont contesté cet argument en avançant que la faiblesse du hadith était telle qu'on ne pouvait y remédier, notamment dans la mesure où il s'oppose aux enseignements coraniques et prophétiques sur le caractère sacré du corps humain. En conclusion, ce hadith ne peut en aucun cas être employé pour justifier les MGF.

\section{Hadith d'Al-Hajjaj ibn Arta}

Il est rapporté dans ce hadith que le prophète (PSL) a dit : "Alkhitaanu (traduit par "circoncision") est sunna pour les hommes et un honneur (makrumah) pour les femmes ». À première vue, c'est-à-dire avant de vérifier son authenticité, le hadith offre deux interprétations. Selon les partisans des MGF, la circoncision est sunna (un acte facultatif) pour les hommes et makrumah (un acte honorable) pour les femmes. Selon les adversaires de la pratique, ce hadith signifie que la circoncision est sunna pour les hommes et que lorsqu'une femme est mariée à un homme circoncis (c'est-à-dire rituellement propre), c'est un honneur pour elle. Cela ne signifie pas que c'est un honneur de soumettre la femme elle-même à la circoncision.

Indépendamment des deux interprétations ci-dessus, le hadith a été classé comme dhaeef (faible) et ne peut être utilisé comme preuve à l'appui des MGF. Par ailleurs, le mot khitaan, qui figure dans le texte original en arabe, est souvent interprété par les partisans de la pratique comme signifiant la circoncision en général (c'est-à-dire à la fois masculine et féminine),

\footnotetext{
${ }^{29}$ Ce terme a été laissé dans sa forme originale en raison de ses diverses significations (expliquées plus loin dans le paragraphe).

${ }^{30}$ Awn al-Maabud fi sharh sunnan Abi Daud, vol. 14, p. 122 à 126.
} 
mais il désigne en fait strictement la circoncision masculine ${ }^{31}$. En arabe, les MGF sont appelées khifaad ${ }^{32}$.

En supposant que le hadith soit authentique, ce qui n'est pas le cas, c'est la seconde interprétation qui serait correcte. Par ailleurs, dans l'interprétation privilégiée par les partisans, sunna est employé en référence à la circoncision masculine et non aux MGF, qui sont simplement désignées comme un acte honorable. Ainsi, l'argument selon lequel les MGF sont sunna n'est pas étayé par ce hadith.

En outre, ce hadith a été conservé par Ahmad et Al-Baihaqqy. Or, d'après les experts en hadith, l'un des narrateurs de la chaîne, Al-Hajjaaj bin Artaa ${ }^{33}$, est muddalis (malhonnête). L'identité de la personne qui a réellement raconté le hadith est également controversée. AlBaihaqqy affirme que ce hadith est dhaeef (faible). Dans son commentaire relatif à Ihyaa Uloom al-Deen' (le renouveau des sciences religieuses) par Al-Ghazaliy, Zainul-Abedeen AlIraqui constate : "En plus des défauts relevés par d'autres savants au sujet de ce hadith, certains pensent qu'il n'est pas valable (baatil), qu'il constitue une invention (maudhuu) et un mensonge (makdhuub) en plus d'être faible (dhaeef) ».

En conclusion, ce hadith a été déclaré faible et non authentique et, pour toutes ces raisons, il ne remplit pas, aux yeux des experts, les critères requis pour être considéré comme un fondement authentique pour justifier les MGF. Un hadith présentant de telles insuffisances ne peut servir de base à aucun acte dans l'Islam, surtout pas lorsque cet acte porte atteinte à la santé et au bien-être d'une personne, alors même qu'aucune MGF n'est mentionnée dans le hadith.

\section{Hadith d'Abdallah ibn Umar}

Ce hadith $^{34}$ dit que les femmes de Al-Ansar (les résidentes de Médine ${ }^{35}$ ) ont été exhortées à pratiquer les MGF. Cependant, il a été déclaré faible par les savants comme Ash-Shaukany car la chaîne de ses transmetteurs comprend Mindal ibn Ali, qui est considéré comme un faible narrateur, et ibn Addy qui est encore plus faible ${ }^{36}$. Ce hadith ne peut être utilisé pour justifier les $\mathrm{MGF}^{37}$.

\section{Hadith d'Aïcha (RA)}

Dans ce hadith, on raconte que le prophète (PSL) a dit : « si les deux parties circoncises (alkhitaanani) se rencontrent (il-tiqaa), un bain rituel (ghusl) est obligatoire ». Dans un autre récit, Aïcha $\left(\mathrm{RA}^{38}\right)$ a rapporté ainsi les paroles du prophète (PSL) : «s'il se place entre ses quatre membres (de la femme), alors les deux parties circoncises se touchent (mass) et un ghusl devient obligatoire » (Sahih Muslim et Tirmidhy ${ }^{39}$ ). Ce hadith est authentique dans

\footnotetext{
${ }^{31}$ Voir Nail-al-autwar, vol. 1, p. 182.

${ }^{32}$ Dictionary of Islamic Legal Terminologie (Dictionnaire de terminologie juridique islamique); Cheikh Ali

Hashim as-Siraj, (2002), FGM/C is female infanticide (traduction), p. 26 à 29.

${ }^{33}$ Voir Nail-al-autwar, vol. 1, p. 183.

${ }^{34}$ Cité dans les séries d'ahadith faibles de Al-Banny, vol. 1.

${ }^{35}$ La ville vers laquelle le prophète (PSL) a émigré à partir de La Mecque et où il a fondé le premier état islamique.

${ }^{36}$ Les spécialistes de la science des ahadith utilisent une série de critères pour déclarer qu'un narrateur est faible, malhonnête ou non fiable.

${ }^{37}$ Voir Nail-al-autwar, vol. 1, p. 183.

${ }^{38}$ Radi-Allahu Anha : que Allah soit satisfait d'elle : voir glossaire des termes.

${ }^{39}$ Deux des livres/recueils les plus fiables des traditions (ahadith) du prophète (PSL).
} 
toutes ses versions et figure dans les chapitres relatifs à la tohara (propreté rituelle) dans tous les livres du fiqh (jurisprudence islamique) ${ }^{40}$.

Les partisans considèrent ce hadith comme l'une des plus fortes justifications des MGF dans l'Islam. Ils pensent que le terme khitaan signifie circoncision en général et donc que khitaanani signifie deux circoncisions, celle de l'homme et celle de la femme. Le hadith traite du rapport sexuel (déduit des mots mass, c'est-à-dire toucher, et iltiqaa c'est-à-dire rencontrer) et puisque le seul rapport sexuel accepté par l'Islam est hétérosexuel, il en découle (pour les partisans) que l'organe féminin est également circoncis, ce qui justifie les MGF.

Des savants ont examiné objectivement ce hadith et ont conclu que bien qu'il soit authentique, il ne peut constituer un fondement justifiant les MGF pour les raisons suivantes :

a. Le hadith se trouve dans le chapitre sur la tohara (purification rituelle ou propreté) dans tous les livres de jurisprudence islamique (fiqh) et les collections de ahadith. Cela signifie que le hadith parle de purification et non de circoncision. Le message du hadith est qu'il est obligatoire de prendre un bain rituel après un rapport sexuel.

b. Les termes employés dans le hadith, khitaanani (littéralement, deux circoncisions masculines), il-tiqaa (littéralement rencontrer) et mass (littéralement toucher), sont des majaaz (métaphores) où khitaanani désigne métaphoriquement les organes sexuels masculin et féminin, mais en utilisant la description de celui qui est tenu d'être circoncis, c'est-à-dire l'organe masculin, et les deux termes de il-tiqaa et de mass décrivent métaphoriquement le rapport sexuel.

c. Par conséquent, il est erroné d'opter pour une interprétation littérale du hadith, selon laquelle un bain rituel devient obligatoire en cas de simple rencontre ou contact entre les organes sexuels masculins et féminins. Ici, les mots « rencontrer/toucher » sont à comprendre dans le contexte de la jurisprudence islamique. Le fait de prendre un bain rituel après un rapport sexuel ne devient obligatoire que si l'une ou les deux situations suivantes se présentent :

- En cas de pénétration. Abu Huraira (RA) a raconté que le prophète (PSL) a dit : «si l'homme s'installe entre les quatre membres de la femme et la pénètre alors un ghusl (bain rituel) est obligatoire, qu'il éjacule ou non ... (Sahib Muslim).

- En cas d'éjaculation. Abi Saiid a raconté que le prophète (PSL) avait dit : «l'eau avec l'eau... » (Sahib Muslim). C'est la raison pour laquelle il est obligatoire de prendre un bain après un rêve érotique ayant déclenché un écoulement de sperme ou de fluide vaginal. Ceci a été raconté dans le hadith d'Ummu-Salama (RA) où une femme appelée Ummu-Suleim a demandé au prophète (PSL) si une femme ayant fait un tel rêve érotique devait ou non prendre un bain rituel et il a répondu : «Naam (oui) » (Sahih al-Boukhari et Muslim).

d. Par ailleurs, il existe un consensus selon lequel le bain est toujours obligatoire, que les deux époux soient ou non circoncis, afin qu'ils se purifient pour le culte (ibada) si l'un ou les deux évènements ci-dessus se sont produits.

\footnotetext{
${ }^{40}$ Voir Nail-al-autwar, vol. 1, p. 332, Fiqhi Sunna, vol. 1, p. 60.
} 
e. En outre, dans la langue arabe, le terme khitaan désigne strictement la circoncision masculine tandis que les MGF sont décrites par le terme de khifaadh, comme nous l'avons expliqué plus haut. Le terme de khitaanani, bien qu'il soit double, n'est pas une preuve en faveur des MGF car l'emploi d'un mot ou d'une qualité pour désigner deux personnes ou deux choses différentes est un style linguistique acceptable en arabe ${ }^{41}$. Dans ce cas khitaanani désigne les organes masculin et féminin, mais ils sont différents en matière de circoncision, c'est-à-dire que l'organe masculin est circoncis, tandis que l'organe féminin ne l'est pas. Le terme employé décrit la caractéristique la plus répandue ou marquant, c'est-à-dire la circoncision masculine. D'autres exemples d'un tel usage sont notamment les suivants :

i. Al-umareyn... (les deux Umar) pour désigner les deux proches compagnons du prophète (Abu bakr et Umar). Le prophète (PSL) a appelé ces deux compagnons «Umareyn », alors qu'ils étaient deux individus distincts.

ii. Al-Bahreyn (deux mers) pour désigner la mer (bahr) et le fleuve (nahr)

iii. Alqamareyn... (les deux lunes) pour désigner le soleil (al-Shams) et la lune (alqamar).

iv. Al-ishaeyn (les deux ishas) en référence aux prières $i s h a^{42}$ et maghrib ${ }^{43}$.

v. Al-aswadeyn (les deux choses noires) pour désigner l'eau et les dattes.

En conclusion, ce hadith ne peut servir de base aux MGF car il porte sur la purification rituelle (tohara) et non sur la circoncision. En outre, le radical d'origine est khitaan, qui est la circoncision masculine et prend aussi la forme de khitaanani en raison du style de la langue arabe, et non parce qu'il désigne littéralement deux circoncisions. De plus, cet élément est corroboré par le fait qu'il n'existe pas de preuve de l'existence de MGF dans le foyer du prophète.

\section{Hadith d'Abu Huraira}

Dans ce hadith, on raconte que le prophète (PSL) a dit : «Les pratiques conformes à la nature primordiale (fitra) à observer par les Musulmans sont au nombre de cinq. Il s'agit de : la circoncision (al khitaan), se raser les poils pubiens, se tailler la moustache, se couper les ongles et s'épiler les aisselles » (Al-Boukhari, Muslim, Abu Daud, An-Nasai, Ahmad et AtTirmidhy ${ }^{44}$ ).

Ce hadith figure également dans le chapitre consacré à la tohara (propreté rituelle), dans tous les livres de jurisprudence islamique (fiqh) et dans les collections de ahadith. Les partisans d'un fondement religieux des MGF s'appuient fortement là-dessus. Le hadith est authentique, mais il ne constitue en aucun cas une preuve que les MGF sont une pratique islamique pour les raisons suivantes :

a. Les cinq actes naturels évoqués dans le hadith ne s'appliquent pas de la même manière aux hommes et aux femmes. Ceux qui s'appliquent aux deux sont : raser les poils pubiens, couper les ongles et épiler les poils sous les aisselles. L'entretien de la moustache et khitaan sont propres aux hommes puisque les femmes ne portent

\footnotetext{
${ }^{41}$ Dans la langue arabe, les mots dérivés par l'addition du suffixe ani expriment l'idée d'un dualisme (khitaansingulier; khitaanani-double), le terme principal employé étant le nom ou le trait distinctif prédominant, ou le plus fort ou celui qui est facile à prononcer. Voir Muhammad Selim El-Awa, p. 8.

${ }^{42}$ Isha est la prière nocturne obligatoire prononcée à partir de $20 \mathrm{~h} 00$.

${ }^{43}$ Maghrib est la prière du soir obligatoire qui commence vers 18 h 30 et se termine vers 19 h 00 .

${ }^{44} \mathrm{Six}$ recueils de traditions authentiques (sahih sita).
} 
naturellement pas de moustache et que le mot khitaan désigne la circoncision masculine, comme nous l'avons expliqué plus haut.

b. Le khitaan mentionné dans le hadith est la circoncision masculine recommandée par le Coran dans le verset cité précédemment : «...puis Nous t'avons révélé [Oh Mahomet] : "suis la religion d'Abraham" » (Coran : $16: 123)$, qui réfère à la circoncision masculine. Les partisans des MGF estiment cependant que ce mot signifie la circoncision en général, tant pour les hommes que pour les femmes.

c. La sunna du prophète (PSL) recommande la circoncision masculine et non les MGF. Il n'aurait pas contredit ses propres actes en ordonnant aux Musulmans de se conformer aux MGF alors que lui-même ne l'a pas fait. Si les MGF constituaient l'un des rites islamiques, il aurait été le premier à les respecter lui-même, tout comme il a respecté la circoncision masculine et enjoint les autres de faire de même.

Cette analyse permet de tirer les conclusions suivantes :

- Ce hadith authentique ne peut être employé pour justifier les MGF.

- Les MGF ne font pas partie des pratiques naturelles que les Musulmans sont tenus de respecter.

Par conséquent, il est clair qu'il n'existe pas de sunna authentique et pertinente en faveur des MGF ; ainsi, il est erroné de les associer aux pratiques du prophète Mahomet (PSL) et même de tout autre prophète. Et surtout, les MGF ne sont pas étayées par les actes des prophètes Mahomet et Abraham (PBUT ${ }^{45}$ ), et il n'est pas concevable que le prophète Mahomet (PSL) ordonne quelque chose qu'il ne pratiquait pas lui-même. Il n'existe aucune preuve démontrant que le prophète (PSL) ait soumis l'une de ses filles ou épouses à des MGF, de même qu'il n'existe aucune preuve démontrant que les épouses et les filles de ses compagnons étaient excisées. Si le prophète (PSL) avait approuvé les MGF pour les membres féminins de sa famille, la pratique serait bien connue et répandue parmi tous les Musulmans, tout comme la circoncision masculine - tel qu'on le voit à l'exemple de ses deux petits-fils, al-Hassan et alHussein - est bien connue et répandue parmi les Musulmans. Par ailleurs, si les MGF étaient une obligation religieuse, le prophète (PSL) ne les aurait pas passées sous silence puisque sa mission était de diffuser les enseignements de la religion. Allah (SWT) lui a ordonné d'agir ainsi dans le Coran : "Ô Messager [Mahomet], transmets [le message] qui t'a été descendu de la part de ton Seigneur. Si tu ne le faisais pas, alors tu n'aurais pas communiqué Son message » (Coran : $5: 67)$.

Le prophète (PSL) a fidèlement accompli sa mission de diffusion de la religion et a enseigné les bons comportements islamiques dans tous les aspects de la vie, y compris la manière de faire sa toilette ou de se conduire à table, les relations entre mari et femme et ainsi de suite. Il n'est donc pas logique qu'il se soit tu à propos d'une question qui concerne un organe reproductif humain important.

Il est recommandé aux Musulmans de veiller à ne pas associer le prophète (PSL) à n'importe quel acte ou parole sans vérifier son exactitude et son authenticité. Il leur a fortement déconseillé de faire des déclarations au sujet de ses actes et paroles sans être certains de leur authenticité. Il a dit : «Quiconque fait délibérément de fausses déclarations à mon sujet devra préparer son siège en enfer... » (Al-Boukhari, ahadith $\mathrm{n}^{\circ} 106,109$ et 110).

\footnotetext{
${ }^{45}$ Paix soit sur eux (Peace Be Upon Them, PBUT).
} 


\section{Ijma'a (consensus de savants islamiques)}

Dans la jurisprudence islamique, le terme ijma'a désigne le consensus entre les opinions des savants du moment. D'après ce principe, lorsqu'il est démontré qu'il existe un consensus sur une question ou une pratique religieuse, ce dernier devient un fondement pour étayer la question, dans la mesure où il ne s'oppose pas au Coran ou à une sunna authentique. En l'occurrence, s'il est démontré que les savants ont établi un consensus sur le statut religieux (hukmu Shari'i) des MGF, ce consensus servira de fondement pour les appuyer.

L'examen de tous les textes relatifs à la jurisprudence islamique (figh) montre que les savants n'ont pas de consensus au sujet des MGF. À titre d'exemple, les quatre écoles de pensée expriment les opinions suivantes :

- l'opinion hanafite est que les mutilations génitales sont sunna (acte facultatif) tant pour les femmes que pour les hommes ;

- l'opinion malikite est qu'elles sont wajib (obligatoires) pour les hommes et sunna (facultatives) pour les femmes ;

- l'opinion chaféite est qu'elles sont wajib (obligatoires) tant pour les femmes que pour les hommes ;

- les hanbalites ont deux opinions sur les mutilations génitales :

- elles sont wajib (obligatoires) tant pour les hommes que pour les femmes

- elles sont wajib (obligatoires) pour les hommes et makrumah (honorables) pour les femmes.

D'autres savants ont exprimé différentes opinions sur les tenants et aboutissants des MGF. Par exemple, d'après Ash-Shaukany ${ }^{46}$, un éminent savant musulman, les MGF sont sunna et comprennent tout ce qui peut être appelé « excision ». Al-Mawardy affirme également qu'elles sont sunna et les décrit comme le fait d'exciser la peau qui ressemble à un capuchon ou à la crête d'un coq, juste au-dessus du vagin ${ }^{47}$. Les opinions ci-après sont celles d'autres savants réputés qui défendent la pratique :

1. Dans son recueil de fatwas (jugements), Ibn Taymiya a dit que les MGF étaient islamiques et que la partie excisée était la membrane supérieure qui ressemblait à la crête d'un coq (voir Fatawa, vol. 21, p. 114).

2. D'après le comité permanent sur les fatwas et les recherches effectuées en Arabie saoudite, la circoncision concerne tant les hommes que les femmes, mais elle est wajib pour les hommes et sunna pour les femmes (voir Fatawa, vol. 5, p. 32).

3. L'ouvrage Al-Fatawa Al-Islamia (les verdicts islamiques), vol. 9, p. 3119 à 3125, affirme que les MGF font partie de l'Islam et qu'aucun savant n'a dit qu'elles ne devaient pas être pratiquées sur les femmes au sens du hadith d'Umm-Atiyya. D'après ces savants, rien dans l'Islam n'interdit l'excision des femmes.

D'un autre côté, il existe de nombreuses opinions, exprimées par des institutions et des savants tout aussi influents, qui s'opposent aux MGF.

\footnotetext{
${ }^{46}$ Voir Nail-al-autwar, vol. 1.

${ }^{47}$ Ibid.
} 
1. Cheikh Sayyid Sabiq a affirmé dans son livre Fiqh As-sunna (vol. 1, p. 33), «Les ahadith affirmant la légalité des MGF sont dhaef (faibles) ; aucun d'entre eux n'est sahih (c'est-àdire authentique)».

2. Cheikh Tantawy - «Il existe un consensus parmi les savants selon lequel la circoncision masculine fait partie de la religion. Il existe une preuve que les petits-fils du prophète (PSL), Al-Hassan et Al-Hussein, étaient circoncis. Il n'existe pas de preuve comparable pour les $M G F^{48} \gg$.

3. Cheikh Mohammed Arafa - «Les savants étudient les MGF et leurs effets. À ce jour, la recherche a prouvé que les organes excisés jouent un rôle très important. Leur excision entraîne des complications et, dans certains cas, l'utilisation de drogues. En supposant, pour la commodité du raisonnement, que les ahadith cités pour étayer la pratique soient authentiques, nous resterions quand même confrontés au problème de l'étendue de l'"excision", chose qui n'est pas claire ${ }^{49} »$.

4. Dans son livre Al-Fatawa (les fatwas), sous le titre $F G M / C$ (p. 2 et 3), Cheikh Shaltoot a dit : «Il n'existe pas un seul élément de preuve démontrant qu'elles font partie de la sunna».

5. Cheikh Abubakar Aljazaairy de la mosquée du prophète (PSL) - « Le terme de khitaan mentionné dans le hadith sur les cinq pratiques conformes à la nature s'applique clairement à la circoncision masculine ».

De toute évidence, il n'existe pas de consensus entre les savants musulmans à ce sujet, de sorte qu'il n'y a aucun élément pouvant servir de preuve sur la base d'un ijma 'a, ou consensus de savants, pour étayer les MGF. Néanmoins, les partisans de la pratique ont fait valoir que les savants n'étaient pas divisés sur la question de savoir si la pratique était islamique ou non, mais plutôt sur son statut et sur la question de savoir si elle était wajib (obligatoire), sunna (facultative), mustahab (recommandée) ou makrumah (honorable), ce qui lui donnerait ainsi le statut de mubaah (autorisée). Par ailleurs, ils ont avancé que puisque aucun savant n'avait dit qu'elle était haram (interdite), il était erroné de dire que les MGF n'était pas islamiques, et que cet acte était au moins mubaah (autorisable).

\section{Commentaires}

Un consensus de savants ne constitue pas un fondement en soi et n'est pas indépendant des dispositions du Coran et de la sunna authentique. Alors que le Coran et la sunna authentique sont les sources révélées de l'Islam et sont donc à l'abri des erreurs, l'ijma' $a$ est un effort humain accompli par des savants pour comprendre le texte révélé et l'appliquer aux questions ou situations ayant une incidence sur la communauté. Les opinions des savants ne devraient donc pas être pris au pied de la lettre, mais soumis à une analyse critique à la lumière des sources révélées.

Plusieurs savants réputés ont commenté les opinions favorables aux MGF. Par exemple, Muhammad Salim Al-Awa, dans son livre FGM/C : an Islamic perspective, a dit : «La compréhension qu'ont les savants des textes religieux est un effort intellectuel purement humain. On ne saurait considérer leur interprétation comme le droit divin proprement dit et l'utiliser comme justification religieuse d'un acte. Elle reflète simplement la manière dont le

\footnotetext{
${ }^{48}$ Voir Al-Azhar Magazine, point Jomâdah Al-Awal, 1417 A.H.

${ }^{49}$ Ibid.
} 
savant comprend le texte et l'applique au sujet dans une situation donnée. Un savant n'est pas ma'asoum (infaillible) ${ }^{50} \gg$ puisque dans l'Islam, l'infaillibilité est un attribut propre aux anges et aux prophètes.

Lorsque les opinions d'un savant sont contraires aux dispositions du Coran et de la sunna authentique, elles ne peuvent pas être acceptées. L'opinion d'un savant peut être juste ou fausse, comme l'a lui-même admis Shafi'i, un éminent savant et fondateur de l'une des quatre écoles de pensée islamique, qui a dit : "Mon opinion est juste mais susceptible d'être fausse, et l'opinion des autres est fausse mais susceptible d'être juste ».

\section{Les MGF sont-elles mubaah (autorisables) ?}

Dans la jurisprudence islamique (fiqh), un acte peut être wajib (obligatoire), sunna (facultatif), mubaah (autorisable), makruh (abhorré) ou haram (interdit). Mubaah désigne un acte légal ou autorisable qui, lorsqu'il est commis ou omis ne constitue ni une vertu, ni un péché. Dans leur effort pour donner aux MGF un fondement religieux, certains partisans sont d'avis qu'elles sont mubaah ou autorisables, en avançant qu'il n'existe pas de disposition explicite qui interdise la pratique, ni dans le Coran ni dans la sunna, et qu'aucun des savants n'a affirmé qu'elle était haram (interdite ou abhorrée).

Les actes dits mubaah ou autorisés doivent remplir certaines conditions, notamment les suivantes :

i. Ils ne sont pas contraires aux dispositions du Coran et de la sunna authentique. Les MGF sont clairement en contradiction avec les règles qui interdisent d'altérer le caractère sacré du corps humain en modifiant la création et en causant un préjudice ; par conséquent, elles ne peuvent être mubaah.

ii. Ils ne causent pas de préjudice à l'individu ou à la société ; un acte néfaste cesse d'être mubaah. Les préjudices causés par tous les types de MGF ont été prouvés sur le plan médical. Il est possible que les savants qui les avaient qualifiées de mubaah ne fussent pas conscients de ces méfaits, peut-être en raison des limites des connaissances scientifiques à l'époque où le verdict a été rendu. Et il existe un principe islamique selon lequel, dès lors qu'une personne a connaissance du caractère condamnable d'un acte, elle doit cesser immédiatement de le pratiquer, car aucun mal ne peut être justifié par son ancienneté. De nos jours, il existe suffisamment de preuves scientifiques démontrant que les MGF sont préjudiciables et ne devraient donc pas être autorisées.

iii. Aucune action pratiquée sur le corps humain ne peut être mubaah car d'après la règle générale de l'Islam, il est illégal d'altérer le corps humain de quelque manière que ce soit en l'absence de preuve évidente de la nécessité de cet acte (al-aslu fii jismul insaan attahrim).

iv. Les MGF qualifiées de sunna (mubaah) sont considérées comme «modérées » ou « minimes ». Mais dans l'Islam, si un acte est haram (interdit), il l'est dans sa totalité et aucune version modérée ou minime n'est autorisée. De même qu'il est haram de boire de la bière, qu'il s'agisse d'une petite gorgée, d'un verre ou d'une caisse entière, il est haram de verser du sang innocent, que ce soit par une piqûre d'épingle, une légère incision ou une ablation d'organe.

\footnotetext{
${ }^{50}$ Muhammad Salim El-Awa, FGM/C from an Islamic Perspective, p. 10.
} 
v. Les partisans ont employé le principe de mubaah (c'est-à-dire al-aslu fil ashiyaai al $i b a h a$ - choses autorisables en règle générale) pour conclure que les MGF sont autorisables (mubaah). Néanmoins, ce principe ne s'applique qu'aux choses ou aux actes destinés à l'usage des êtres humains, sauf preuve du contraire, c'est-à-dire sauf démonstration de leur interdiction. Il ne s'applique pas au corps humain puisque la règle générale interdit tout acte pratiqué sur le corps, à moins qu'il existe une preuve qui l'autorise (voir iii ci-dessus).

Ainsi, les MGF ne peuvent pas être mubaah puisqu'elles causent un préjudice et modifient la création humaine. Il est erroné de dire que les MGF ne sont pas interdites, car les dispositions du Coran et de la sunna authentique condamnent explicitement tout acte qui cause un préjudice au corps humain ou modifie d'une quelconque manière la création d'Allah (SWT) sans justification. Ces interdictions sont applicables aux MGF.

\section{Qiyas (déduction par analogie)}

Il s'agit d'un principe de jurisprudence islamique qui implique la comparaison d'actes ou de situations présentant des caractéristiques communes ( $\left.I^{\prime} l l a^{51}\right)$, lorsque l'un des actes a été jugé et pas l'autre et que le verdict du premier est appliqué au second. Un exemple serait une comparaison entre l'alcool et l'héroïne. L'alcool a été explicitement déclaré illégal, mais il n'existe pas de condamnation directe de l'héroïne. Cependant, les deux substances ont une caractéristique commune, celle d'être enivrante, et comme l'alcool a été déclaré illégal parce qu'il est enivrant, l'héroïne est aussi déclarée illégale dans l'Islam parce qu'elle a un effet enivrant comme l'alcool. Le prophète Mahomet (PSL) a dit : "Tout alcool est une substance enivrante et toutes les substances enivrantes sont interdites $»\left(\right.$ Sahih Boukhari $\left.{ }^{52}\right)$.

Les partisans ont fait valoir que les MGF étaient islamiques parce, selon le principe du qiyas, la pratique est comparable à la circoncision masculine. Cependant, une telle comparaison entre la circoncision masculine et les MGF est impossible à établir puisque les deux pratiques ne partagent pas de caractéristiques ni de causes (I'llat) communes. La comparaison entre les deux révèle les éléments suivants :

a. La circoncision masculine a un fondement indiscutable dans l'Islam et constitue de ce fait un acte islamique. Les MGF n'ont aucun fondement comparable et ne peuvent être qualifiées d'islamiques.

b. Les MGF sont en contraires aux enseignements de l'Islam concernant le caractère sacré du corps humain alors que la circoncision masculine est conforme aux préceptes religieux.

c. La circoncision masculine n'est pas controversée ; elle est universellement pratiquée par tous les musulmans, tandis que les MGF sont controversées et sont pratiquées seulement par certaines communautés musulmanes.

d. La partie excisée chez les hommes est une peau dépourvue de fonction essentielle alors que chez les femmes, ce sont des organes fonctionnels qui sont soit retirés, soit altérés.

e. L'étendue de la partie retirée lors de la circoncision masculine est clairement définie et universellement acceptée par tous les musulmans. En revanche, dans le cas des MGF, l'étendue et la partie à exciser ne sont pas définies, mais laissées à la discrétion d'individus, d'où les variations observées d'une communauté à l'autre. Si les MGF étaient islamiques, l'étendue de l'excision serait clairement définie, comme c'est le cas de la circoncision masculine.

\footnotetext{
${ }^{51}$ Voir glossaire des termes.

${ }^{52}$ Al-Boukhari, vol. 5, Hadith $n^{\circ} 623$.
} 
f. La circoncision masculine présente des avantages prouvés (religieux et médicaux), tandis que les MGF n'en procurent aucun ; elles entraînent uniquement des préjudices évidents.

\title{
Conclusion
}

L'examen de toutes les sources fondamentales révèle clairement qu'il n'existe pas de fondement authentique des MGF dans l'Islam. Pas un seul verset du Coran ne peut servir à les appuyer ; au contraire, de nombreux versets condamnent la pratique. Il n'existe aucune tradition authentique de la sunna du prophète Mahomet (PSL) qui étaye les MGF. Les savants ont des opinions divergentes sur la pratique ; certains l'ont qualifiée de wajib (obligatoire) alors que d'autres la considèrent comme makrumah (un acte honorable). Le principe du qiyas n'est pas applicable dans la mesure où il n'y a pas de caractéristique commune entre la circoncision masculine, qui dispose d'un fondement dans l'Islam, et les MGF. Ainsi, il est clair que les MGF peuvent uniquement être considérées comme une pratique culturelle, et non religieuse.

\section{Les enseignements islamiques qui s'opposent aux MGF}

En plus de l'absence d'une base étayant les MGF dans les sources fondamentales de la pensée islamique, bon nombre d'enseignements islamiques fondamentaux sont contredits par la pratique. Ceux-ci constituent des arguments de poids, susceptibles d'être employés pour s'opposer à la pratique.

\section{Les MGF sont contraires aux objectifs de la charia}

La charia islamique, qui énonce les principes directeurs de l'Islam, a pour but de préserver les éléments suivants :
1. Religion
2. Vie
3. Intellect
4. Progéniture
5. Richesse.

Tout ce qui porte atteinte à l'un ou l'autre de ces objectifs est interdit dans l'Islam. Les MGF ont un impact négatif direct sur tous les cinq objectifs de la charia. Il est par exemple connu que des jeunes filles et des femmes sont mortes après l'opération, en raison de complications entraînées par MGF, tant immédiatement qu'à long terme. Étant donné qu'il n'existe aucune justification de la pratique, elle est considérée comme une violation directe des objectifs de la charia. Elle est donc non islamique et quiconque la pratique commet un péché.

\begin{abstract}
Allah (SWT) condamne ceux qui modifient Sa création
Parmi les nombreuses justifications des MGF figure la croyance selon laquelle la pratique embellirait les organes génitaux féminins. Des personnes interrogées dans le cadre de l'une des études réalisées dans la province nord-orientale ont dit par exemple : "J'exciserai ma fille car je ne veux pas que les gens disent qu'elle est vide, je veux qu'elle soit magnifique et que sa chose [soit] brillante comme un miroir » et «vous savez, ce clitoris pousse avec le corps de la jeune fille. Ils ne veulent pas voir ce truc, il aura l'air anormal, et il deviendra laid ${ }^{53} »$.
\end{abstract}

\footnotetext{
${ }^{53}$ Jaldesa G.W., Askew I., Njue C., Wanjiru M., Female genital cutting among the Somali of Kenya and management of its complications, Population Council : Nairobi, Kenya.
} 
Ceci montre que beaucoup des partisans des MGF croient qu'à moins d'être excisés, les organes génitaux féminins s'allongent et s'enlaidissent.

Cette croyance est contraire aux enseignements de l'Islam, selon lesquels Allah (SWT) a dit : «Nous avons certes créé l'homme dans la forme la plus parfaite» (Coran : 95 : 4). Ainsi, la beauté d'un corps humain doit être laissée comme Allah l'a créée, à moins qu'il n'y ait un fondement authentique autorisant une intervention.

D'après le Coran, tout acte équivalant à modifier la création d'Allah est considéré comme l'œuvre de Satan. Les MGF consistent à exciser des organes fonctionnels et sains et à modifier l'aspect des organes génitaux féminins en l'absence de tout fondement religieux, ce qu'Allah (SWT) condamne fermement. Il a même condamné ceux qui altèrent les organes des animaux et a maudit Iblis (Satan) qui a dit : «Certes, je ne manquerai pas de les égarer, je leur donnerai de faux espoirs, je leur commanderai, et ils fendront les oreilles aux bestiaux; je leur commanderai, et ils altèreront la création d'Allah... »(Coran : 4 : 119). Dans le même verset, Allah (SWT) met en garde ceux qui imitent Satan dans de tels actes et dit : «...quiconque prend le Diable pour allié au lieu d'Allah sera, certes, voué à une perte évidente »(Coran : $4: 119)$.

Le prophète (PSL) a également dit : "Allah a maudit ceux qui se liment les dents, celles qui allongent leurs cheveux par des ajouts et ceux qui modifient la création de Dieu ${ }^{54}{ }$.

\section{Causer un préjudice est illégal}

L'Islam condamne toutes les pratiques néfastes et destructrices, comme par exemple l'infanticide féminin. Allah (SWT) dit dans le Coran : " et qu'on demandera à la fillette enterrée vivante pour quel péché elle a été tuée » (Coran : 81 : 8-9). Il a été médicalement prouvé que les MGF sont nocives, même dans leur forme la plus modérée, car elles altèrent les fonctions normales des organes génitaux féminins. Chacun des organes concernés par la pratique (clitoris, petites lèvres, grandes lèvres) a une fonction spécifique à remplir et toute intervention sur eux affecte leur fonctionnement et leur finalité.

Les principes directeurs suivant sont énoncés dans la charia :

- La dharar wala dhirar (ne cause pas de préjudice et ne retourne pas un préjudice $)^{55}$. Les MGF sont une pratique néfaste qu'il convient d'éviter. Allah dit dans le Coran : «......et ne vous jetez pas par vos propres mains dans la destruction » (2 : 195).

- Si une action entraîne à la fois des bénéfices et des préjudices, elle est autorisée si les premiers dépassent les seconds. Pour la circoncision masculine, par exemple, l'excision de la peau, la douleur dont elle s'accompagne ainsi que le fait de verser du sang peuvent être considérés comme néfastes. Cependant, il a été prouvé que la circoncision masculine est une pratique religieuse et que les bénéfices qui en résultent sont également significatifs. Ces bénéfices sont à la fois d'ordre religieux (la circoncision masculine renforce la propreté) et médical (la circoncision masculine peut réduire les risques de cancer du pénis et de transmission du $\mathrm{VIH}^{56}$ ). En revanche, les MGF produisent exactement le contraire, en altérant la propreté religieuse et en causant un préjudice prouvé.

\footnotetext{
${ }^{54}$ Voir commentaire de Sahih Muslim par An-Nawawi, vol. 13, p. 102.

${ }^{55}$ Majalat al-ahkam al-a'daliya (journal sur les principes juridiques), article 19, p. 18.

${ }^{56} \mathrm{http}$ ://wikipedia.org/wiki/medical analysis of circumcision\#penile-cancer
} 
- Adh-dhararu la yuzaalu bi mithlihi ${ }^{57}$ / Adh-dhararul ash-shadu yuzaalu bi dhararin $a l$-akhaf ${ }^{58}$ (un préjudice ou un mal n'est pas supprimé ou stoppé par la commission d'un préjudice équivalent ou supérieur). Dans le présent contexte, le préjudice ou le mal présumé par les partisans des MGF est le fait que le clitoris s'allonge et devient laid et malodorant. Le préjudice causé par les MGF dépasse largement les bénéfices perçus.

\section{L'Islam condamne les pratiques culturelles néfastes}

L'une des raisons évoquées à l'appui des MGF est qu'elles constituent une pratique culturelle pour les communautés qui s'y conforment.

« Notre tradition veut qu'une jeune fille soit excisée et cousue... »(femmes mariées, Jogbaru $^{59}$ ).

L'Islam s'abstient de déclarer illégales ou d'interdire toutes les pratiques culturelles. Les Musulmans sont libres de poursuivre leurs cultures et pratiques traditionnelles tant que cellesci ne sont pas contraires aux enseignements de l'Islam. C'est la raison pour laquelle certaines coutumes arabes antérieures à l'Islam ont été conservées alors que d'autres ont été condamnées. De même, les Musulmans de toutes les tribus et communautés sont tenus d'examiner leurs cultures, de poursuivre ce qui est conforme à l'Islam et d'éviter tout ce qui s'y oppose.

Pour pouvoir être maintenue par l'Islam, une pratique culturelle doit remplir deux conditions importantes :

- elle ne doit pas être contraire aux enseignements de l'Islam.

- elle ne doit pas causer de préjudice, ni à l'individu, ni à l'ensemble de la société.

D’après les enseignements islamiques, les pratiques culturelles sont traitées comme suit :

- Les pratiques culturelles conformes aux enseignements de l'Islam sont confirmées comme des pratiques islamiques. À titre d'exemple, l'hospitalité, la chasteté, le respect des anciens et l'aide aux pauvres, qui sont préconisées dans de nombreuses cultures, sont en accord avec l'Islam.

- les pratiques culturelles contraires à l'Islam sont soit modifiées afin qu'elles s'y conforment, soit totalement supprimées (par exemple, la polygamie a été réglementée, l'infanticide féminin a été supprimé $\left.{ }^{60}\right)$.

Les MGF s'opposent aux enseignements islamiques sur le caractère sacré du corps humain et sont donc totalement inacceptables. Elles constituent également une pratique fortement enracinée et nombreux sont les membres de communautés qui disent qu'elles sont pratiquées depuis longtemps et ne peuvent donc pas être stoppées. Certains Somali affirment : «il est difficile pour les Somali d'arrêter la circoncision pharaonique. Elle existe ici et il est difficile de la stopper. Elle va continuer, il faudra beaucoup de temps pour qu'un changement se

\footnotetext{
${ }^{57}$ Majalat al-ahkam al-a'daliya (le journal sur les principes juridiques), article 20, p. 19.

${ }^{58}$ Ibid., article 25, p. 19.

${ }^{59}$ FRONTIERS 2005, FGM/C Baseline Study in Wajir District.

${ }^{60} \mathrm{Il}$ s'agit ici de cultures qui existaient dans l'Arabie pré-islamique.
} 
produise... » et «nous savons que la religion n'est pas d'accord avec nous, mais les gens sont très attachés à la tradition ${ }^{61} »$.

Ceci est contredit par l'enseignement islamique selon lequel le mal ne peut être trop vieux, ce qui signifie que des pratiques anciennes ne se justifient pas lorsqu'elles sont néfastes. À partir du moment où l'on découvre qu'elles ont causé un préjudice, il convient de les arrêter, quelle que soit leur ancienneté. Le Coran mentionne des cas où des communautés ont obstinément défendu leurs mauvaises actions, en affirmant qu'elles poursuivraient ces pratiques parce qu'elles les avaient héritées de leurs ancêtres. Allah (SWT) dit : "Et quand on leur dit : "Suivez ce qu'Allah a fait descendre", ils disent: "Non, mais nous suivrons les coutumes de nos ancêtres"... » (Coran : 2 : 170). Allah (SWT) condamne ce raisonnement et demande dans ce même verset si les gens imiteraient leurs prédécesseurs «... si leurs ancêtres n'avaient rien raisonné et s'ils n'avaient pas été dans la bonne direction ? (Coran : 2 : 170).

Les MGF n'ont aucun fondement religieux ; elles constituent une pratique purement culturelle qui s'oppose aux enseignements islamiques. L'un des enseignements fondamentaux de l'Islam précise qu'en cas de conflit entre la religion et une pratique culturelle, l'Islam prime. Les MGF s'opposent aux enseignements islamiques et il faut y mettre un terme.

Les femmes ont droit à un corps sain et au plaisir sexuel dans leurs relations conjugales L'une des raisons de pratiquer les MGF est la croyance selon laquelle elles maîtrisent le désir sexuel des femmes et des jeunes filles, ce qui les rend chastes. Parmi de nombreuses communautés pratiquant les MGF, une croyance répandue veut qu'une femme non excisée ressente un désir sexuel excessif et par conséquent « «commencera à courir après les hommes à cause de sa sensualité incontrôlable, de son désir sexuel excessif, elle sera très vulnérable, elle n'aura aucune sécurité et finira par perdre son honneur. Les jeunes filles excisées ne chercheront pas un autre homme...» (praticien de $\mathrm{MGF}^{62}$ ).

Les désirs sexuels sont naturels, ce sont des dons divins. L'Islam ne s'oppose pas au sexe, mais donne des orientations sur la manière dont il convient de satisfaire le désir sexuel, en reconnaissant que le mari aussi bien que la femme ont droit au plaisir sexuel. L'Islam n'autorise pas les pratiques qui portent atteinte au plaisir sexuel dans le mariage. Plusieurs dispositions du Coran et de la sunna donnent des indications sur le plaisir sexuel dans le mariage. Les directives du Coran sur les relations sexuelles entre mari et femme ont été interprétées comme incluant les préliminaires. Allah (SWT) dit : "Vos épouses sont pour vous un champ de labour; allez à votre champ comme [et quand] vous le voulez et æuvrez pour vous-mêmes à l'avance... »(Coran : 2 : 223). Le prophète (PSL) a dit : "Lorsqu'un homme a des rapports sexuels avec sa femme, il doit être au même rythme qu'elle. S'il satisfait son désir avant elle, il ne se retirera pas tant qu'elle ne sera pas également satisfaite... » (Hadith d'Anas Ibn Malik) ${ }^{63}$.

Interrogés sur les avantages des MGF, leurs partisans affirment qu'elles diminuent les désirs sexuels des femmes et garantissent ainsi la chasteté. Bien que l'Islam soit favorable à la chasteté, il n'accepte pas la mutilation d'organes comme moyen de la garantir. Tous les types de MGF altèrent le fonctionnement sexuel des femmes et s'opposent ainsi à leur droit de jouir pleinement du plaisir sexuel, qui est un don divin.

\footnotetext{
${ }^{61}$ Ibid.

${ }^{62}$ Jaldesa G.W., Askew I., Njue C., Wanjiru M. 2004. Female genital cutting among the Somali of Kenya and management of its complications. Population Council : Nairobi, Kenya.

${ }^{63}$ Voir Said Sabiq, Fiqhi Sunna, vol. 2, p. 142 et 143.
} 


\section{Ne pas punir l'innocent}

Comme exposé plus haut, de nombreux partisans des MGF croient qu'elles contrôlent les désirs sexuels des jeunes filles et des femmes en limitant leur libido. Cependant, cette pratique n'est pas acceptable dans la mesure où elle revient à punir une personne innocente en prévision de l'éventualité que celle-ci commettrait le crime de fornication ou d'adultère (zinaa). Elle est comparable au fait d'amputer les mains des gens, dans les pays où est appliquée la charia, de crainte qu'ils ne volent plus tard. Il s'agit d'une injustice et nul ne devrait être puni pour un délit qui n'a pas encore été commis. Dans l'Islam, aucun châtiment n'est appliqué avant que soit prouvé qu'un crime a été commis. De plus, il existe des dispositions concernant les peines relatives à l'adultère et à la fornication; la mutilation des organes génitaux n'en fait pas partie. Par ailleurs, c'est le cerveau humain qui contrôle nos actions et en assume la responsabilité, et non les organes. Ainsi, les désirs sont contrôlés par le cerveau, qu'ils soient de nature sexuelle ou autre, et aucun degré de mutilation ne peut garantir la chasteté si le cerveau en décide autrement.

\section{L'éducation islamique des enfants (tarbiyya) garantit la chasteté}

Les partisans de la pratique affirment que les MGF garantissent la chasteté et la droiture morale en contrôlant les désirs sexuels, et les relient ainsi aux exigences islamiques de chasteté et de droiture morale. L'Islam reconnaît que les désirs, qu'ils soient de nature sexuelle ou autre, sont contrôlés par le cerveau et non par le corps. Ainsi, il souligne l'importance d'une bonne éducation (tarbiyya) et d'enseignements moraux pour contrôler la chasteté. Les MGF n'ont aucun rapport avec la discipline ou la droiture morale des enfants ; en effet, les observations effectuées dans des communautés qui les pratiquent montrent que de nombreuses jeunes filles excisées ne parviennent pas à maîtriser leurs désirs et ont des relations sexuelles en dehors du mariage. Par ailleurs, de nombreuses jeunes filles non excisées font preuve de chasteté et de droiture morale. Il est humiliant pour toute femme d'être considérée comme une personne incapable de maitriser ses actions et ses désirs sexuels, et donc d'être obligée de subir une mutilation de ses organes génitaux afin de les contrôler.

Il convient de garder à l'esprit le fait que dans l'Islam, la chasteté est une exigence qui concerne tant les hommes que les femmes, car les femmes ne peuvent pas, à elles seules, maintenir la chasteté dans la communauté. Nul n'a affirmé que la circoncision masculine contrôle le désir sexuel des hommes ; en fait, il est souvent dit qu'elle améliore leur sexualité. Il est ironique de constater que les communautés qui veulent contrôler la sexualité de leurs femmes veulent en même temps renforcer la sexualité de leurs hommes. Tous deux sont tenus d'observer les exigences islamiques de chasteté et de moralité au moyen d'une éducation morale.

Il est important de relever que dans ses enseignements, le prophète (PSL) accorde une grande importance à l'éducation des enfants et à l'environnement dans lequel ils grandissent. Le fait de veiller à ce qu'un enfant reçoive une tarbiyya islamique correcte est une amana (confiance) accordée aux parents, aux tuteurs et à l'ensemble de la société. Le prophète (PSL) dit : "Chacun de vous est gardien, et tout gardien sera interrogé à propos de la charge qui lui a été confiée » ${ }^{64}$.

\footnotetext{
${ }^{64}$ Boukhari et Muslim.
} 
Afin d'assurer que les enfants grandissent en vue d'être chastes et de se comporter moralement, l'Islam enjoint les parents et les tuteurs à mettre l'accent sur la tarbiyya, qui comprend les éléments suivants :

i. Éduquer l'enfant, car l'Islam insiste sur la connaissance. Le prophète (PSL) dit : « La recherche de la connaissance est une obligation pour tous les musulmans ${ }^{65} \gg$. Dans un autre récit il a dit : "Quiconque suit un chemin pour chercher la connaissance, Allah aplanit pour lui le chemin vers Janah (le paradis) ${ }^{66}{ }^{»}$.

ii. Doter l'enfant d'un savoir religieux islamique dès la plus tendre enfance. Lui inculquer la conscience d'Allah (SWT), c'est-à-dire la taqwa, la connaissance de ses droits et de ses devoirs, afin qu'il sache comment entretenir des relations avec le créateur et avec ses frères humains, et distinguer le bien du mal.

iii. Enjoindre l'enfant à faire ce qui est bien et à éviter ce qui est mal.

iv. Enseigner à l'enfant l'importance d'un bon caractère (akhlaq).

v. Encourager l'enfant à observer les actes du culte (ibada) dès son plus jeune âge afin de lui apprendre la discipline et l'obéissance.

vi. Veiller à ce que l'enfant ait de bons amis et le protéger des mauvaises influences.

vii. Toujours rappeler à l'enfant l'importance de la chasteté et de l'honneur, en citant les modèles de personnes chastes et en racontant comment Allah (SWT) sera satisfait d'eux, en citant des exemples comme ceux du prophète Joseph (Yussuf) (AS) et de Marie (Maryam) (RA), la mère du prophète Jésus (Issa) (AS).

viii. Souligner l'importance du code vestimentaire islamique dès la tendre enfance.

\section{L'infibulation affecte la propreté rituelle (tohara)}

Nombreux sont les partisans qui croient que les MGF améliorent la propreté. «Celles qui ne sont pas excisées sont sales, et elles produiront toujours une odeur fétide, alors nous sommes excisées pour rester propres » (femmes récemment mariées, discussions en groupes de réflexion) ${ }^{67}$.

Cependant, la pratique consistant à exciser et infibuler ${ }^{68}$ les jeunes filles et les femmes, adoptée par certaines communautés musulmanes, porte atteinte à leur tohara (propreté rituelle) car les femmes infibulées ne peuvent atteindre la pureté requise par l'Islam après un passage aux toilettes. Au lieu de s'écouler naturellement au-dehors, l'urine traverse lentement la paroi créée par l'infibulation, qui recouvre l'orifice de l'urètre. Il leur est impossible de nettoyer l'espace situé sous cette peau, parce que l'ouverture laissée pour l'écoulement de l'urine et des menstruations est trop étroite. Sans tohara, le culte (ibada) n'est pas autorisé dans l'Islam. Les jeunes filles et les femmes infibulées sont ainsi privées du droit de pratiquer le culte et, par extension, du premier objectif pour lequel elles ont été créées. Allah (SWT) dit : «Je n'ai créé les djinns et les hommes que pour qu'ils M'adorent... » (Coran : 51 : 56).

\section{Les MGF sont une violation des droits humains des femmes et des jeunes filles}

Les droits humains sont des droits divins accordés à tous les êtres humains, du fait de leur appartenance au genre humain. L'Islam prône le respect des droits, de la dignité et du bienêtre de tout être humain. Il a été démontré que les MGF sont une violation des droits des

\footnotetext{
${ }^{65}$ Tirmidhy $\mathrm{n}^{\circ} 74$.

${ }^{66}$ Muslim 1381 dans Riyadhu-as-salihin

${ }^{67}$ Jaldesa G.W., Askew I., Njue C., Wanjiru M.. Female genital cutting among the Somali of Kenya and management of its complications. Population Council : Nairobi, Kenya.

${ }^{68}$ MGF de type III d'après la classification de l'OMS.
} 
femmes et des jeunes filles tels qu'ils sont reconnus par l'Islam. Certains de ces droits compromis par la pratique sont les suivants :

\section{Le droit à la vie}

Les MGF causent un préjudice douloureux et peuvent entraîner la mort, pendant ou après la procédure, par suite d'une infection, par exemple, ou d'une perte de sang. Chose étonnante, certains partisans de la pratique affirment que si la mort survient du fait des MGF, c'est la volonté de Dieu. Mais cet argument n'est pas conforme aux enseignements de l'Islam qui exigent que l'on fasse tout ce qui est humainement possible pour éviter les préjudices. À ce sujet, Allah (SWT) dit dans le Coran, « ...et ne vous jetez pas par vos propres mains dans la destruction $\gg($ Coran : 2 : 195).

\section{Le droit à un corps sain}

L'Islam défend le caractère sacré du corps humain. Nul n'a le droit de mutiler ou de causer une douleur ou un préjudice à un corps humain, que ce soit le sien ou celui d'une autre personne. Aucun parent ni tuteur n'a de droits sur le corps d'une fille, car ils n'en sont pas propriétaires. Les savants religieux doivent jouer leur rôle et enseigner aux parents et aux tuteurs que le fait de prendre soin et d'élever des enfants ne leur confère pas le droit de mutiler leurs corps.

\section{Le droit à une vie saine}

Tout être humain a le droit de vivre une vie sans douleur ni préjudice. Les MGF entraînent de nombreuses complications de santé dues à l'altération du fonctionnement normal du corps de la jeune fille, car les organes touchés comportent de nombreuses terminaisons nerveuses et sont particulièrement sensibles. Les MGF affectent négativement la santé et le bien-être des jeunes filles et des femmes tout au long de leurs vies et constituent une violation de leurs droits.

\section{Le droit au plaisir sexuel}

Les enseignements de l'Islam soulignent qu'une femme, tout comme un homme, a droit au plaisir sexuel dans le mariage. Les MGF affectent les organes de l'appareil génital féminin qui jouent un rôle important dans la sexualité. Le clitoris, par exemple, a essentiellement pour fonction de contribuer à la jouissance sexuelle féminine et son ablation ou altération affecte le fonctionnement sexuel. La pratique constitue une violation du droit des femmes au plaisir sexuel et n'est donc pas acceptable dans l'Islam.

\section{Le droit au culte}

D'après l'Islam, les êtres humains ont été créés pour adorer Allah (SWT) et toute personne a le droit de pratiquer le culte. Tout ce qui l'en empêche est une violation de ce droit fondamental. Le type d'excision couramment pratiqué dans la corne de l'Afrique, notamment parmi les Somali, est la forme la plus extrême (type III), l'infibulation, qui prive la jeune fille ou la femme de la possibilité de se nettoyer après être allée aux toilettes, ou lors des menstruations, et la prive ainsi du droit d'atteindre l'état de propreté rituelle (tohara) nécessaire pour pratiquer le culte.

\section{Le droit de faire un choix}

Dans certaines communautés comme celle des Somali, les filles sont soumises aux MGF à un très jeune âge : entre quatre et dix ans et parfois plus tôt. À cet âge, la fillette n'a aucune capacité légale de choisir ou de donner un consentement, notamment sur les interventions affectant son corps. De plus, elle est rarement consultée et il est présumé que le parent ou le 
tuteur a le droit de décider ce qu'il convient de lui faire. D'après la charia islamique, ceci est une grave violation du droit de la fillette à faire un choix dans sa vie.

Les exemples susmentionnés montrent clairement que les MGF ne sont pas conformes aux droits humains soutenus par l'Islam. L'Islam défend la protection et la promotion des droits humains et ne peut admettre aucune pratique qui y soit contraire. Ces violations sont criminelles et donc punissables dans le monde d'ici-bas et dans l'au-delà.

\section{Des efforts sont nécessaires pour corriger les maux dans la communauté}

Certains savants et membres des communautés qui sont convaincus que les MGF n'ont aucun fondement islamique reconnaissent la nécessité de les arrêter, mais disent que seul Allah (SWT) peut sauver la communauté et mettre fin à la pratique. Si l'Islam exige de faire confiance à Allah (SWT) et de prier pour Son intervention, il demande également de faire ce qui est humainement possible pour corriger un mal. On ne peut pas se contenter de souhaiter la disparition du problème, car Allah (SWT) dit : "En vérité, Allah ne modifie point l'état d'un peuple, tant que les [individus qui le composent] ne modifient pas ce qui est en euxmêmes... »(Coran : $13: 11)$.

\section{Les savants religieux ont la responsabilité de corriger les maux dans la communauté}

Les savants religieux sont les personnes qualifiées pour jouer un rôle moteur sur les questions se rapportant aux enseignements de l'Islam. En effet, ils ont l'immense avantage de savoir lire, comprendre et interpréter les textes religieux. Cependant, ils n'ont pas été en mesure de combattre la pratique pour plusieurs raisons, la principale étant que leur information inadéquate sur les organes génitaux féminins, leurs fonctions et les effets des MGF sur la santé et le bien-être des jeunes filles et des femmes. Cela s'explique par le secret entourant la pratique ainsi que par une «gêne » à aborder la question. Peu de savants souhaitent débattre de ce sujet, car ils le considèrent comme honteux et dégradant et n'y voient pas un problème prioritaire.

Pour pouvoir appréhender l'ampleur du problème et jouer leur rôle légitime, les savants doivent consulter des experts. L'Islam recommande aux individus de solliciter des informations auprès de spécialistes pour être en mesure de prendre des décisions en connaissance de cause. Allah (SWT) dit dans le Coran : « ...Demandez donc aux gens du rappel si vous ne savez pas » (Coran : $16: 43$ ). Dans le contexte des MGF, une fois qu'un médecin, en sa qualité de spécialiste du corps humain, donne un avis sur le préjudice causé par la pratique, les savants religieux sont tenus d'appliquer la loi divine à cette connaissance. D'après le verset ci-dessus, même si les savants religieux disposent du savoir leur permettant de rendre des verdicts sur le caractère islamique ou non islamique des MGF, ils doivent solliciter l'expertise des médecins pour comprendre les fonctions des organes génitaux féminins et les effets de toute intervention sur ces derniers. Ainsi ils comprendront pleinement les implications des MGF au moment de rendre leur verdict. Les savants religieux doivent s'appuyer sur les avis spécialisés des médecins pour éclairer leurs verdicts, et s'abstenir de fonder leurs vues sur l'opinion publique. Ils ont le devoir d'utiliser ce savoir et d'appliquer la loi divine pertinente, c'est-à-dire les enseignements de l'Islam relatifs aux pratiques néfastes, et de déclarer les MGF non islamiques.

\section{Ne pas succomber aux pressions communautaires}

Bon nombre de savants appartenant aux communautés musulmanes qui pratiquent les MGF savent que celles-ci sont dépourvues de fondement religieux et sont contraires aux enseignements de la religion. Cependant, ils ne sont pas encore parvenus à prendre 
publiquement position pour condamner la pratique. Ceci est principalement dû aux pressions exercées sur eux par les communautés et à leur crainte de perdre le respect des fidèles. Ils savent que la pratique a été associée à tort à l'Islam, ils possèdent des connaissances approfondies de l'Islam, mais ils répugnent à endosser leur responsabilité de décourager la pratique.

L'Islam recommande à chaque croyant de ne point succomber aux pressions communautaires au prix de désobéir à Allah (SWT). Lorsqu'un conflit se fait jour entre les enseignements divins d'Allah (SWT) et les intérêts de la communauté, les premiers doivent primer. Allah (SWT) dit dans le Coran : "Il n'appartient pas à un croyant ou à une croyante, une fois qu'Allah et Son messager ont décidé d'une chose, d'avoir encore le choix dans leur façon d'agir. Et quiconque désobéit à Allah et à Son messager, s'est égaré certes, d'un égarement évident » (Coran : 33 : 36). Dans l'Islam, un mal ou un préjudice doit être supprimé ou arrêté : adhararu yuzaalu. Le Coran affirme : "Vous êtes la meilleure communauté qu'on ait fait surgir pour les hommes. Vous ordonnez le convenable, interdisez le blâmable et croyez à Allah...»(Coran : 3 : 110). Ce précepte est appuyé également par l'enseignement du prophète (PSL) selon lequel tout élément néfaste est à supprimer par tous les moyens possibles. Le prophète (PSL) dit : "Quiconque parmi vous voit un mal doit l'extirper de ses mains, mais s'il ne peut le faire, il l'enlève avec les mots de sa bouche, et s'il ne peut le faire, il la déteste avec son cour. C'est le minimum de la foi ${ }^{69}{ }$.

\section{L'origine des MGF n'est pas islamique}

L'origine exacte des MGF est inconnue, même de certaines des communautés qui les pratiquent couramment ${ }^{70}$. Leur forme la plus radicale, l'infibulation, pratiquée par la communauté somali, est appelée circoncision pharaonique. Bien que le nom suggère que la pratique a débuté dans l'Égypte ancienne, sous les pharaons, rien ne permet d'affirmer avec certitude qu'elles ont commencé dans ce pays ou dans une autre partie de l'Afrique. Quoiqu'il en soit, on raconte que l'un des pharaons ${ }^{71}$ fut informé par des devins qu'un enfant de sexe masculin verrait le jour parmi les Israélites et mettrait fin à son règne. Il ordonna alors de tuer tous les garçons israélites et d'infibuler toutes les femmes israélites de sorte que celles-ci aient besoin d'une sage-femme pour accoucher, d'où le terme de « circoncision pharaonique ». Ainsi le pharaon était assuré d'être informé de la naissance de chaque garçon pour le faire tuer. Si l'histoire est vraie, cela veut dire que la pratique est antérieure à l'Islam, et les Musulmans n'ont aucun avantage à imiter une telle pratique.

Une autre origine historique invoquée par les partisans des MGF est que la pratique remonterait à Sarah et Hajar, les épouses du prophète Abraham (AS). Sarah était restée stérile pendant très longtemps. Lorsque Hajar, la seconde épouse, eut un fils, Sarah jura de la tuer. Il est rapporté que le prophète Abraham (AS) dit à Sarah qu'il n'était pas juste de tuer Hajar, mais que pour annuler le serment, elle pouvait lui percer les oreilles et exciser une partie de ses organes génitaux. Cette histoire n'a aucune authenticité. Au mieux, on peut dire qu'elle constitue un mythe montrant que la première femme excisée était Hajar et que l'exciseuse était Sarah. Et au cas où l'histoire serait vraie, l'excision aurait été effectuée à titre de punition et en raison d'une jalousie, et non pas comme une tradition. De plus, s'il s'agissait d'une pratique courante, qui aurait excisé Sarah ? Ceci ne fait que confirmer la nature non islamique de la pratique.

\footnotetext{
${ }^{69}$ Sahih Muslim Hadith $\mathrm{n}^{\circ} 16$.

${ }^{70}$ FRONTIERS 2005, FGM/C Baseline Study in Wajir District.

${ }^{71}$ Le pharaon de l'époque du prophète Musa (Moïse).
} 
Les MGF sont pratiquées par les Musulmans et les non Musulmans pour différentes raisons. Pour certains, c'est un rite de passage, pour d'autres, c'est le signe d'une identité culturelle, mais la plupart des communautés musulmanes croient que les MGF sont une coutume islamique. Or, elles ne sont pas pratiquées dans un certain nombre de pays musulmans, tels que l'Iran, l'Arabie saoudite, la Libye, l'Algérie et le Pakistan, pour n'en nommer que quelques-uns. Au Kenya, certaines communautés non musulmanes appliquent les MGF et certaines communautés musulmanes non somali ne le font pas. Ainsi, la pratique ne se limite pas aux musulmans et ne peut pas être considérée comme islamique.

\section{Conclusions}

Les enseignements de l'Islam fournissent des preuves écrasantes attestant que les MGF ne sont pas une pratique religieuse et que l'Islam les condamne. Néanmoins, elles sont toujours défendues par certaines communautés musulmanes comme une pratique religieuse, et cette question a soulevé une vive controverse parmi les musulmans. Les éléments de preuve cités à partir du Coran, des sunna (traditions) authentiques, des ijma'a (consensus de savants) et des qiyas (déductions par analogie) pour étayer les MGF justifient la circoncision masculine, qui est confirmée par le mode de vie (milat) des deux prophètes, Abraham et Mahomet (PBUT). Rien ne prouve cependant que les femmes de l'époque étaient également excisées durant leur vie. Les MGF sont une coutume culturelle pratiquée dans certaines communautés, et il est erroné d'associer l'Islam à une pratique aussi néfaste. L'Islam a clairement énoncé des dispositions en faveur de la protection des droits humains fondamentaux et défend le caractère sacré du corps humain. Toute pratique qui viole ces droits et cause un préjudice au corps humain sans aucune justification est interdite.

Les savants religieux, de par le respect et l'influence dont ils bénéficient dans leurs communautés, sont les personnes les mieux placées pour dissocier les MGF de l'Islam et faire connaître leurs conséquences néfastes. Ils sont responsables de corriger les maux de la communauté et doivent relever le défi d'aborder le problème des MGF dans leurs propres communautés ainsi que dans l'ensemble de l'umma (communauté) musulmane. Afin de comprendre pleinement la pratique et ses effets, il est important que les savants religieux collaborent avec les médecins afin de rendre des verdicts fondés sur des faits scientifiques.

En plus des savants et des autres personnes faisant autorité, c'est à chaque individu qu'il incombe de mettre fin à cette pratique. Chaque personne doit prendre sciemment la décision d'abandonner les MGF dans sa famille. Ainsi, les parents ont l'obligation de protéger leurs filles ainsi que les jeunes filles confiées à leurs soins. Chaque personne devra répondre de ses actes et doit donc remplir ses obligations et s'y préparer. 


\section{GLOSSAIRE DES CONCEPTS/TERMES}

MGF : Ce terme est employé dans la présente brochure pour désigner la pratique des mutilations génitales féminines (MGF) telle qu'elle est définie par l'OMS ${ }^{72}$ et d'autres organisations internationales. Cependant, les connaissances acquises de l'expérience et de la recherche montrent que la plupart des membres des communautés perçoivent l'excision de la pointe ou d'une petite partie du clitoris comme une pratique religieuse musulmane appelée sunna. Ils considèrent également que le terme de MGF se rapporte spécifiquement au type III (infibulation), généralement connu sous le nom de firouni en somali, ce qui signifie pharaonique. Afin d'éviter toute confusion et de prévenir l'impression erronée que la présente brochure ne viserait qu'à dissocier l'infibulation de l'Islam, le terme de MGF est employé ici pour désigner tous les types de mutilations génitales, quelle que soit la terminologie locale.

La pratique : désigne la pratique des mutilations génitales féminines.

Partisans : désigne ceux qui soutiennent et défendent la pratique.

Hadith : communication orale du prophète Mahomet (PSL).

Ahadith : pluriel de hadith.

Fiqh : étude de la science du droit islamique (jurisprudence islamique).

Qiyas : principe juridique de déduction par analogie. Il est employé dans la jurisprudence islamique pour rendre un verdict relatif à une question qui n'a pas encore fait l'objet d'un verdict, mais présente une caractéristique commune avec une autre question dotée d'un verdict ; elle constitue une source secondaire d'enseignements islamiques.

I'llat : cause ou caractéristique commune employée lors de l'application du principe du qiyas.

Charia : système de normes islamiques régissant l'existence.

Sunna : signifie littéralement chemin ou voie, mais le terme est employé sur dans le contexte religieux pour désigner les paroles, les actes ou les approbations du prophète Mahomet (PSL). Dans la jurisprudence islamique $(f i q h)$ il y a des actes facultatifs ou recommandés. La sunna constitue une source primaire des enseignements de l'Islam.

Ijma'a : consensus d'opinions de savants élaborés après la mort du prophète Mahomet (PSL) au sujet de diverses questions islamiques. Il constitue l'une des sources secondaires des enseignements de 1'Islam.

Subhannahu Wa Taala (SWT) : le Très-Haut ; il s'agit de l'un des attributs d'Allah prononcés après avoir mentionné Son nom, en reconnaissance de Sa puissance.

Radhi-Allahu Anhu (lui) / Anha (elle) (RA) : que Allah (SWT) soit satisfait de lui / d'elle, utilisé respectivement après le nom des compagnons/compagnes du prophète Mahomet (PSL) dans l'Islam.

\footnotetext{
${ }^{72}$ OMS. 2008. Éliminer les mutilations sexuelles féminines. Déclaration interinstitutions. OMS : Genève.
} 
Paix Soit sur Lui (PSL) : supplique en faveur du prophète Mahomet, employée après son nom pour solliciter pour lui la paix et la bénédiction d'Allah (SWT).

Alleihi Salam (AS) : que la paix soit avec lui, employé après avoir mentionné le nom d'un prophète, comme supplique en sa faveur et pour solliciter pour lui la paix d'Allah (SWT). 
POUR PLUS D'INFORMATIONS, VEUILLEZ CONTACTER :

FRONTIERS IN REPRODUCTIVE HEALTH

Population Council

4301 Connecticut Avenue, N. W.

Suite 280

Washington, D.C. 20008

USA

Téléphone : 202-237-9400

Fax : $\quad$ 202-237-8410

E-mail : $\quad$ frontiers@pcdc.org

Site Web : www.popcouncil.org

AFRIQUE

Population Council Regional Office

P.O. Box 17643

Nairobi

Kenya

Téléphone : 254-2-2713480/1/2/3

Fax : $\quad$ 254-2-2713479

E-mail : publications@popcouncil.org

\section{ASIE ET PROCHE-ORIENT}

Population Council Regional Office

Ground Floor, Zone 5A

India Habitat Center

Lodi Road

New Delhi 110003

Inde

Téléphone : 91-11-461-0913

Fax : $\quad$ 91-11-464\2903

E-mail : $\quad$ frontiers@pcindia.org

AMÉRIQUE LATINE ET CARAÏBES

Population Council Regional Office

Escondida 110

Villa Coyoacan

04000 Mexico, D.F.

Mexique

Téléphone : 52-5-659-8537

Fax : 52-5-554-1226

E-mail : disemina@popcouncil.org.mx 


\section{(2) Population Council}

Le Population Council est une organisation internationale, non gouvernementale et à but non lucratif. Sa mission est d'améliorer le bien-être et la santé reproductive des générations actuelles et à venir dans le monde entier et d'assurer un équilibre humain, équitable et durable entre les gens et les ressources. Le Council réalise des recherches biomédicales, de sciences sociales et de santé publique et renforce les capacités de recherche dans des pays en développement. Créé en 1952, le Council est dirigé par un conseil d'administration international. Son siège à New York soutient un réseau mondial de bureaux régionaux et nationaux.

\section{FRONTIERS \\ IN REPRODUCTIVE HEALTH}

Le programme FRONTIERS est financé par le bureau de la population de l'Agence américaine pour le développement international (UNITED STATES AGENCY FOR INTERNATIONAL DEVELOPMENT, USAID) en vertu des termes de l'accord de coopération n HRN-A-00-98-00012-00.

Le document original à été traduit en français grâce au soutien financier du projet suprarégional «Abandon des mutilations génitales féminines» de la Gesellschaft für Internationale Zusammenarbeit $(\mathrm{GIZ}) \mathrm{GmbH}$, commandée par le ministère fédéral de la Coopération économique et du Développement (BMZ). 